\title{
Modeling of Community and Hospital-acquired Methicillin-resistant Staphylococcus Aureus Transmission in Hospital Settings
}

\author{
F. B. Agusto \\ ${ }^{\dagger}$ Department of Ecology and Evolutionary Biology, University of Kansas, Lawrence, KS, 66045, USA \\ ${ }^{\ddagger}$ Department of Statistics, University of Georgia, Athens, GA, 30602, USA \\ ${ }^{* *}$ Department of Mathematics and Statistics, Austin Peay State University, Clarksville, TN, 37044, USA
}

Received: 24 October 2015, accepted: 16 November 2015, published: 22 November 2015

\begin{abstract}
In this paper we developed both deterministic and stochastic models of community- and hospital-acquired methicillin-resistant staphylococcus aureus transmission (MRSA) to quantify their interactions in a hospital settings. The disease-free equilibrium of the model is locally-asymptotically stable whenever the associated reproduction number is less than unity. The disease persists in the community whenever the reproduction number is greater than unity. Although our stochastic model evolves on an unbounded state space, we show it is positive recurrent. The result obtained from the sensitivity analysis using the deterministic model indicates that the dominant parameters are the hand washing compliance rate, the health-care workers decolonization rate, environmental contamination rate, the admission rates into the hospital, isolation rate of patients with CA-MRSA and isolation rate of patients with HA-MRSA, the transmission probabilities of CA- and HA-MRSA per contact with healthcare workers and transmission probability of healthcare workers per contact with patients. Numerical simulations of the deterministic model obtained from using the dominate parameters as combination of control strategies such as low-, moderateand high-effectiveness control strategies show that
\end{abstract}

disease prevalence among the hospital patients and the bacterial in the hospital environment can be controlled by moderate- and high-effectiveness control strategies. However, for health-care workers the disease prevalence can only be effectively controlled by the high-effectiveness control strategy.

Key words: community-associated MRSA, hospitalacquired MRSA, disease prevalence, sensitivity analysis, continuous-time Markov chain, forward Kolmogorov equations

\section{INTRODUCTION}

Methicillin-resistant Staphylococcus aureus (MRSA) is a gram-positive bacterium; a strain of staphylococcus aureus bacteria that is resistant to commonly used antibiotics used in the treatment of ordinary staphylococcus aureus infections. More than 70 percent of the bacteria that cause hospital-acquired MRSA (HA-MRSA) infections are resistant to at least one of the drugs most commonly used in treatment of infections. HAMRSA infections mostly occur among hospital 
patients or residents of nursing homes and dialysis centers and other health care settings. According to the Centers for Disease Control (CDC) more than two million people every year acquire some form of clinically significant antibiotic-resistant infection, with at least 23,000 deaths as a result [4]. In particular, CDC estimated 80,461 invasive MRSA infections in 2011 with 11,285 related deaths. An unknown but much higher number of less severe infections occurred in both the community and in health-care settings [4]. The economic impact of antibiotic resistance on the U.S. economy is estimated to be as high as \$20 billion in excess direct health-care costs, with additional costs to society for lost productivity as high as \$35 billion a year (2008 dollars) [4].

MRSA causes substantial morbidity and mortality and is endemic in hospitals and nursing homes. Health-care-associated MRSA (HA-MRSA) infections are typically associated with individuals who have had invasive procedures or indwelling medical devices, such as intravenous tubing or artificial joints and/or antimicrobial therapy. Communityassociated MRSA (CA-MRSA) infections, on the other hand, arise in otherwise healthy individuals without such risk factors. CA-MRSA infections were first described by Savoralatz et al. in 1982 among intravenous drug abusers [41], [42] and later reported in the early 1990s in patients without prior health-care contact in Western Australia and New Zealand as well as in American children in the late 1990s [14], [23], [46]. The CA-MRSA strains were genetically distinct from traditional health-care-associated MRSA [34], [44] and are more virulent and rapidly spreading than the traditional hospital-associated MRSA strains [14]. Five CA-MRSA lineages have been found worldwide: ST1-IV (USA400); ST8-IV (USA300); ST30-IV (Pacific/Oceania); ST59-IV and V (USA1000, Taiwan) and ST80-IV (European) [16]. CA-MRSA outbreaks in hospitals have been reported since 2003 from North America, Germany, Israel, Switzerland, Greece and the UK, often in units such as paediatrics and obstetrics where the preva- lence of HA-MRSA is low [38].

A number of mathematical modeling studies have been carried out on HA-MRSA and CA-MRSA, to quantify the potential impact of the burden of the epidemic (see [6], [9], [12], [13], [26], [39], [44]). For instance, Chamchod and Ruan [6] developed and analyzed deterministic and stochastic models to investigate the transmission dynamics of MRSA and to determine factors that influence the prevalence of MRSA infection when antibiotics are given to patients to treat or prevent infections with either MRSA itself or other bacterial pathogens. Cooper [9] presented a mathematical model of both hospital and community reservoirs of MRSA colonization to explain substantial increases in HA-MRSA and CA-MRSA despite rigorous control policies. They showed how the timing of the intervention, the level of resource provision, and chance combine to determine whether control measures succeed or fail. D'Agata et al. [12] developed a deterministic model to characterize the transmission dynamics of HA-MRSA and CAMRSA in the hospital setting and to quantify the emergence of co-colonization with both strains. Their results show that the state of co-colonization becomes endemic over time and that typically there is no competitive exclusion of either strain. $\mathrm{D}^{\prime}$ Agata et al. [13] developed a deterministic model to characterize the factors contributing to the replacement of hospital-acquired MRSA with CA-MRSA and to quantify the effectiveness of interventions aimed at limiting the spread of CAMRSA in health care settings. Their results suggest that CA-MRSA will become the dominant MRSA strain in hospitals and health care facilities. Pressley [39] formulated a model of HA-MRSA and CA-MRSA first under the assumption that patients cannot be co-colonized by the two strains. Their results show that competitive exclusion occurs between HA-MRSA and CA-MRSA strains. They further extended the model to include cocolonization of patients with the two strains and the extended model hardly exhibits competitive exclusion. Skov [44] presented a mathematical model 
showing the influence of MRSA transmission in the community on the prevalence of MRSA in hospitals.

Some studies indicate the progression of MRSA may be largely explained by contaminated healthcare workers coming in direct contact with patients [9], [35]. Chamchod and Ruan [6] studied the transmission dynamics of health-care workers in nursing homes and found that minimizing contact with the residents and following proper handwashing procedures reduced transmission of the bacteria. Milazzo [35] showed certain measures such as improving hand hygiene compliance, barrier precaution policies, and effective staff management, can be taken to control the bacteria. Milazzo further suggested relocating contaminated patients to separate wards may prevent MRSA from spreading quickly throughout the hospital [35]. Chow [7] found isolation has the potential to effectively control the progression of the disease. Thus, in this study, we develop new deterministic and stochastic models for the spread of CA-MRSA and HA-MRSA in hospital settings and include the health-care worker interactions with patients and contamination of the environment a feature that is absent in the models in [6], [7], [9], [12], [35], [39]. Furthermore, we allow for isolation of infected patients another feature absent in the models in [6], [9], [12], [35], [39]. Our objective in this study is to determine the model parameters with the most dominant impact on the spread of MRSA using the deterministic model conditions for elimination of the disease in the hospital setting. Using these parameters and a combination low-, moderate- and high-effectiveness control strategies, we determine if disease prevalence can be controlled in the hospital setting.

The current paper is organized as follows: In Section $[\Pi$ we describe the community-and hospitalacquired MRSA transmission model, followed by basic qualitative analysis in Section III. The system is modeled as a continuous time Markov chain in Section IV. The uncertainty and sensitivity analysis of the model is investigated in Section $\mathrm{V}$ And in Section VI, we investigate the impact of basic control measures defined as low-, moderateand high-effectiveness control strategies.

\section{Model Formulation}

The model is formulated as follows: the total patient population in the hospital $N_{H}(t)$ is split into mutually-exclusive sub-populations of individuals who are uncolonized $\left(U_{P}\right)$, colonized with CAMRSA strain $\left(C_{C H}\right)$, colonized with HA-MRSA strain $\left(C_{H}\right)$, infected with CA-MRSA $\left(I_{C H}\right)$, infected with HA-MRSA $\left(I_{H}\right)$, or isolated $(Q)$, so that

$$
\begin{aligned}
N_{H}(t)= & U_{P}(t)+C_{C H}(t)+I_{C H}(t)+C_{H}(t) \\
& +I_{H}(t)+Q(t)
\end{aligned}
$$

Similarly, the total population of health-care workers at time $t$, denoted by $N_{W}(t)$, is split into mutually-exclusive sub-populations of uncontaminated $\left(U_{W}\right)$ and contaminated $\left(C_{W}\right)$ health-care workers, so that

$$
N_{W}(t)=U_{W}(t)+C_{W}(t)
$$

We assume throughout that $N_{W}(t)$ remains constant in time. In addition, the variable $E_{H}$ models the overall contamination level of the hospital environment and can be thought of as the total amount of bacterial contamination on the floors, walls, doorknobs, etc.

The hospital-and-community-acquired MRSA transmission model in the hospital setting is given by the following system of non-linear differential 
F. B. Agusto et al., Modeling of Community and Hospital-acquired Methicillin-resistant ...

equations:

$$
\begin{aligned}
\frac{d U_{P}}{d t}= & \Pi_{P}\left(1-\pi_{C C}-\pi_{I C}-\pi_{C H}-\pi_{I H}\right) \\
& -\left(\lambda_{C H}+\lambda_{H}\right) U_{P}-\left(\mu_{H}+\alpha_{P}\right) U_{P} \\
& +\tau_{C H} C_{C H}+\tau_{H} C_{H}
\end{aligned}
$$$$
\frac{d C_{C H}}{d t}=\pi_{C C} \Pi_{P}+\lambda_{C H} U_{P}+\gamma_{C H} I_{C H}
$$$$
-\left(\tau_{C H}+\sigma_{C H}+\omega_{C C H}\right.
$$$$
\left.+\mu_{H}+\alpha_{C}\right) C_{C H}
$$$$
\frac{d I_{C H}}{d t}=\pi_{I C} \Pi_{P}+\sigma_{C H} C_{C H}
$$$$
-\left(\gamma_{C H}+\omega_{I C H}+\mu_{H}+\delta_{C H}\right) I_{C H}
$$$$
\frac{d C_{H}}{d t}=\pi_{C H} \Pi_{P}+\lambda_{H} U_{P}+\gamma_{H} I_{H}-
$$$$
\left(\tau_{H}+\sigma_{H}+\omega_{C H}+\mu_{H}+\alpha_{H}\right) C_{H}
$$

$$
\begin{aligned}
\frac{d I_{H}}{d t}= & \pi_{I H} \Pi_{P}+\sigma_{H} C_{H} \\
& -\left(\gamma_{H}+\omega_{I H}+\mu_{H}+\delta_{I H}\right) I_{H} \\
\frac{d Q}{d t}= & \omega_{C C H} C_{C H}+\omega_{I C H} I_{C H}+\omega_{C H} C_{H} \\
& +\omega_{I H} I_{H}-\left(\mu_{H}+\delta_{Q}+\alpha_{Q}\right) Q
\end{aligned}
$$$$
\frac{d U_{W}}{d t}=-\lambda_{W} U_{W}+\tau_{W} C_{W}
$$$$
\frac{d C_{W}}{d t}=\lambda_{W} U_{W}-\tau_{W} C_{W}
$$$$
\frac{d E_{H}}{d t}=\varepsilon_{W} C_{W}-\rho_{E H} E_{H} .
$$

The flow diagram is depicted in Figure 1 and the associated state variables and parameters are described in Table I]

In system (2.1), the parameter $\Pi_{P}$ is the total rate at which patients are admitted into the hospital (with units of number per day), and the parameters $\pi_{C C}, \pi_{I C}, \pi_{C H}, \pi_{I H}$ are the proportions of admitted patients colonized with CA-MRSA $\left(C_{C H}\right)$, infected with CA-MRSA $\left(I_{C H}\right)$, colonized with HAMRSA $\left(C_{H}\right)$ or infected with HA-MRSA $\left(I_{H}\right)$, respectively. The parameters $\tau_{C H}, \tau_{H}$ and $\tau_{W}$ are decolonization rates of colonized individuals and health-care workers. The parameters $\lambda_{C H}, \lambda_{H}$ and $\lambda_{W}$ are infection rates of uncolonized patients and health-care workers (following effective contact with colonized patients, health-care workers and contaminated environment). The infection rates $\lambda_{C H}, \lambda_{H}$ and $\lambda_{W}$ are defined as

$$
\begin{gathered}
\lambda_{C H}=\frac{\beta_{C}\left(C_{W}+\zeta_{1} E_{H}\right)(1-\eta)}{N_{W}} \\
\lambda_{H}=\frac{\beta_{H}\left(C_{W}+\zeta_{2} E_{H}\right)(1-\eta)}{N_{W}}
\end{gathered}
$$

and

$$
\begin{aligned}
\lambda_{W}= & \left(\beta _ { W } \left[\eta_{1}\left(C_{C H}+C_{H}\right)+I_{C H}+I_{H}\right.\right. \\
& \left.\left.+\zeta_{3} E_{H}\right](1-\eta)\right) /\left(N_{W}\right),
\end{aligned}
$$

where $\beta_{C}$ and $\beta_{H}$ are CA-MRSA and HA-MRSA are infection probabilities of uncolonized patients per contact with health-care workers and the contaminated environment, while $\beta_{W}$ is the infection probability of uncontaminated health-care workers per contact with colonized or infected patients and the contaminated environment. The parameters $\zeta_{1}, \zeta_{2}, \zeta_{3} \in[0,1]$ are modification parameters that control for the effect of the contaminated environment on the infection rates [8], [37], [49]. For example, $\zeta_{2}$ near 1 (0) implies a high (low) contribution of the environmental contamination to the HA-MRSA infection rate. The parameter $\eta \in[0,1]$ models the health-care workers' daily hand-washing compliance rate. For example, when 
$\eta=1$ there is perfect hand-washing compliance each day and the infection rates become $0 ; \eta=0$ coincides with no hand-washing. The unitless parameter $\eta_{1} \in[0,1]$ controls for the contribution of the total number of colonized patients to the rate at which hospital workers become colonized. The parameters $\alpha_{P}, \alpha_{C}, \alpha_{H}$ and $\alpha_{Q}$ are the discharge rates from the hospital and $\mu_{H}$ is the natural death rate of patients. The parameters $\gamma_{C H}$ and $\gamma_{H}$ represent the recovery rates due to effective treatment, $\sigma_{C H}$ and $\sigma_{H}$ represent the progression rates of colonized individuals into the infectious class $\left(I_{C H}, I_{H}\right)$, and $\delta_{C H}, \delta_{I H}$ and $\delta_{Q}$ are MRSA induced death rates. The parameters $\omega_{C_{C H}}, \omega_{I_{C H}}, \omega_{C H}$ and $\omega_{I H}$ correspond to the isolation rates of the colonized individuals with CA-MRSA and HA-MRSA strains respectively. A simplifying assumption we have made for this model is that the health-care workers are the only ones contributing to environmental contamination, since they can move around in the hospital and are responsible for the sanitation of the hospital. To this end, the parameter $\varepsilon_{W}$ is the rate at which a single contaminated health-care worker contaminates the hospital environment. Also, $\rho_{E H}$ is the contaminant decay rate. Another simplifying assumption we have made is that the patients are not co-colonized with the two strains of MRSA. We are aware that this is not what holds in reality and as part of our future work, we intend to include the co-colonization of patients with the two MRSA strains.

The model 2.1 is an extension of numerous published MRSA transmission models (e.g., those in [6], [7], [9], [12], [35], [39]) by (inter alia):

(a) including environmental contamination by the health-care workers (this was not considered in [6], [7], [9], [12], [35], [39]);

(b) including isolation of infected patients to prevent the further spread of the bacteria (this was not considered in [6], [9], [12], [35], [39]).

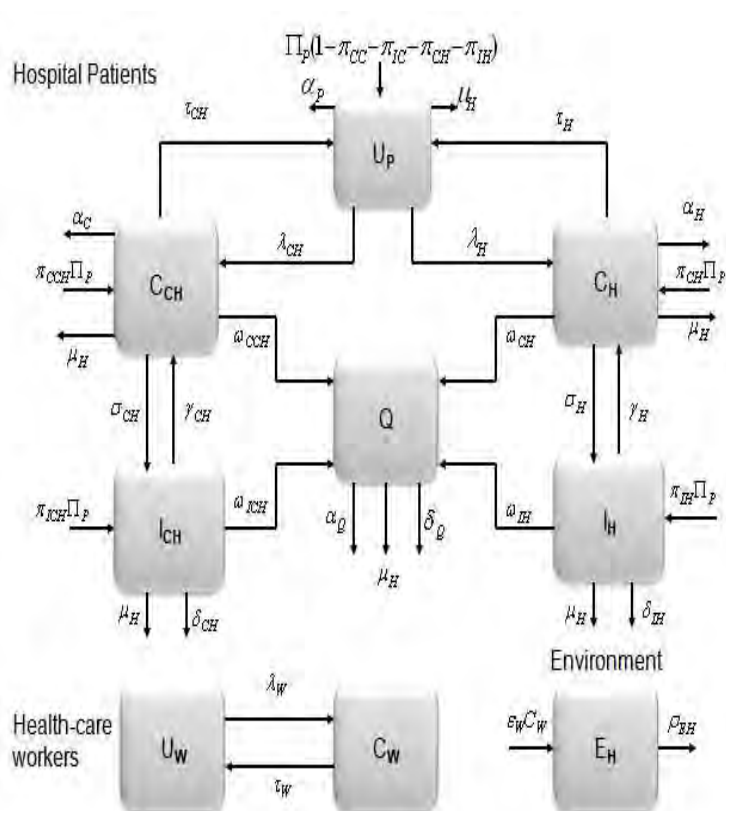

Fig. 1. Flow diagram of model 2.1

\section{Qualitative Analysis of The Model}

In this section, the conditions for the existence and stability of the equilibria of the model 2.1 in the hospital setting are explored.

\section{A. Basic Qualitative Properties}

1) Positivity and boundedness of solutions: For the model (2.1) to be epidemiologically meaningful, all its state variables have to be non-negative for all time (that is, the solutions of the model system 2.1 with non-negative initial data will remain non-negative for all time $t>0$ ). The following result can be established (using, for instance, the method in Appendix A of [45]).

Lemma 1. Let the initial data $U_{P}(0) \geq$ $0, C_{C H}(0) \geq 0, I_{C H}(0) \geq 0, C_{H}(0) \geq$ $0, I_{H}(0) \geq 0, Q(0) \geq 0, U_{W}(0) \geq$ $0, C_{W}(0) \geq 0$. Then the solutions $\left(U_{P}(t), C_{C H}(t), I_{C H}(t), C_{H}(t), I_{H}(t), Q(t)\right.$, 


\begin{tabular}{ll}
\hline \hline Variable & Description \\
\hline \hline & \\
$U_{P}(t)$ & Population of uncolonized patients \\
$C_{C H}(t)$ & Population of colonized with CA-MRSA strain \\
$C_{H}(t)$ & Population of colonized with HA-MRSA strain \\
$I_{C H}(t)$ & Population of infected with CA-MRSA \\
$I_{H}(t)$ & Population of infected with HA-MRSA \\
$Q(t)$ & Population of isolated patients \\
$U_{W}(t)$ & Population of uncontaminated health-care workers \\
$C_{W}(t)$ & Population of contaminated health-care workers \\
$E_{H}(t)$ & Contaminated hospital environment \\
\hline \hline
\end{tabular}

$\begin{array}{ll}\beta_{C} & \text { Transmission probability of CA-MRSA per contact } \\ \beta_{H} & \text { Transmission probability of HA-MRSA per contact } \\ \beta_{W} & \text { Transmission probability of health-care workers } p \\ \eta & \text { Hand washing compliance rate } \\ \eta_{1}, \zeta_{1}, \zeta_{2}, \zeta_{3} & \text { Modification parameters } \\ \Pi_{P} & \text { Number of hospital admission per day } \\ \pi_{C C} & \text { Admission rate into colonized CA-MRSA class } \\ \pi_{I C} & \text { Admission rate into infected CA-MRSA class } \\ \pi_{C H} & \text { Admission rate into colonized HA-MRSA class } \\ \pi_{I H} & \text { Admission rate into infected HA-MRSA class } \\ \tau_{C H} & \text { Decolonization rate from } C_{C H} \text { to the } U_{P} \text { class } \\ \tau_{H} & \text { Decolonization rate from } C_{H} \text { to the } U_{P} \text { class } \\ \tau_{W} & \text { Decontamination rate from } C_{W} \text { to the } U_{W} \text { class } \\ \mu_{H} & \text { Natural death rate } \\ \alpha_{P} & \text { Discharge rate for the uncolonized class } \\ \alpha_{H} & \text { Discharge rate for the colonized HA-MRSA class } \\ \alpha_{C} & \text { Discharge rate for the colonized CA-MRSA class } \\ \alpha_{Q} & \text { Discharge rate for the isolated class } \\ \gamma_{C H} & \text { Recovery rate from the infected CA-MRSA class } \\ \gamma_{H} & \text { Recovery rate from the infected HA-MRSA class } \\ \sigma_{C H} & \text { Progression rate to the infected CA-MRSA class } \\ \sigma_{H} & \text { Progression rate to the infected HA-MRSA class } \\ \omega_{C C H} & \text { Isolation rate of } C C H \text { class } \\ \omega_{I C H} & \text { Isolation rate of } I_{C H} \text { class } \\ \omega_{C H} & \text { Isolation rate of } C_{H} \text { class } \\ \omega_{I H} & \text { Isolation rate of } I_{H} \text { class } \\ \delta_{C H} & \text { Mortality rate of the infected CA-MRSA class } \\ \delta_{H} & \text { Mortality rate of the infected HA-MRSA class } \\ \delta_{Q} & \text { Environmental contamination rate } \\ \varepsilon_{W} & \\ \rho_{E H} & \\ & \text { Environmental contaminant decay rate } \\ & \end{array}$

TABLE I

VARIABLES AND PARAMETER DESCRIPTIONS OF MODEL 2.1. 
$\left.U_{W}(t), C_{W}(t), E_{H}(t)\right)$ of the model (2.1) are non-negative for all $t>0$. Furthermore, the region

$$
\begin{array}{r}
\Omega=\left\{\left(U_{P}, C_{C H}, I_{C H}, C_{H}, I_{H}, Q, U_{W}, C_{W}, E_{H}\right)\right. \\
\in \mathbb{R}_{+}^{9}: N_{P}(t) \leq \frac{\Pi_{P}}{\mu_{H}}, N_{W}(t)=K_{W}, E_{H}(t) \\
\left.\leq \frac{\varepsilon_{W} K_{W}}{\rho_{E H}}\right\}
\end{array}
$$

is positively-invariant for the model (2.1) with non-negative initial conditions in $\mathbb{R}_{+}^{9}$.

The prove of Lemma 1 Appendix A.

\section{B. Local Stability of the Disease-Free Equilibrium (DFE)}

In this section, the conditions for the existence and stability of the equilibria of the model 2.1 is explored.

The model (2.1) has a DFE, given by

$$
\begin{array}{r}
\mathcal{E}_{H C}=\left(U_{P}^{*}, C_{C H}^{*}, I_{C H}^{*}, C_{H}^{*}, I_{H}^{*}, Q^{*}, U_{W}^{*}, C_{W}^{*}, E_{H}^{*}\right) \\
=\left(\frac{\Pi_{P}}{\mu_{H}+\alpha_{P}}, 0,0,0,0,0, N_{W}^{*}, 0,0\right) .
\end{array}
$$

Using the notations in [48], and rearranging the infected compartments in the order: $C_{C H}, I_{C H}, C_{H}$, $I_{H}, Q, C_{W}$ and $E_{H}$, the matrices $F$ and $V$ for the new infection terms and the remaining transfer terms are, respectively, given by,

$$
F=\left(\begin{array}{cccc}
0 & 0 & 0 & 0 \\
0 & 0 & 0 & 0 \\
0 & 0 & 0 & 0 \\
0 & 0 & 0 & 0 \\
0 & 0 & 0 & 0 \\
\beta_{W} \eta_{1}(1-\eta) & \beta_{W}(1-\eta) & \beta_{W} \eta_{1}(1-\eta) & \beta_{W}(1-\eta) \\
0 & 0 & 0 & 0 \\
& 0 & \frac{\beta_{C}(1-\eta) U_{P}^{*}}{U_{W}^{*}} & \frac{\beta_{C} \zeta_{1}(1-\eta) U_{P}^{*}}{U_{W}^{*}} \\
& 0 & 0 & 0 \\
& 0 & \frac{\beta_{H}(1-\eta) U_{P}^{*}}{U_{W}^{*}} & \frac{\beta_{H} \zeta_{2}(1-\eta) U_{P}^{*}}{U_{W}^{*}} \\
& 0 & 0 & 0 \\
& 0 & 0 & 0 \\
& 0 & 0 & \beta_{W} \zeta_{3}(1-\eta) \\
& 0 & 0 & 0
\end{array}\right)
$$

and

$$
V=\left(\begin{array}{ccccccc}
k_{1} & -\gamma_{C H} & 0 & 0 & 0 & 0 & 0 \\
-\sigma_{C H} & k_{2} & 0 & 0 & 0 & 0 & 0 \\
0 & 0 & k_{3} & -\gamma_{H} & 0 & 0 & 0 \\
0 & 0 & -\sigma_{H} & k_{4} & 0 & 0 & 0 \\
-\omega_{C C H} & -\omega_{I C H} & -\omega_{C H} & -\omega_{I H} & k_{5} & 0 & 0 \\
0 & 0 & 0 & 0 & 0 & \tau_{W} & 0 \\
0 & 0 & 0 & 0 & 0 & -\varepsilon_{W} & \rho_{E H}
\end{array}\right)
$$

where, $k_{1}=\tau_{C H}+\sigma_{C H}+\omega_{C C H}+\mu_{H}+\alpha_{C}, k_{2}=$ $\gamma_{C H}+\omega_{I C H}+\mu_{H}+\delta_{C H}, k_{3}=\tau_{H}+\sigma_{H}+\omega_{C H}+\mu_{H}+$ $\alpha_{H}, k_{4}=\gamma_{H}+\omega_{I H}+\mu_{H}+\delta_{H}$ and $K_{5}=\alpha_{Q}+\mu_{H}+\delta_{Q}$.

It follows that the basic reproduction number of the model 2.1), denoted by $\mathcal{R}_{H C}$, is given by

$$
\begin{aligned}
& \mathcal{R}_{H C}=\rho\left(F V^{-1}\right)=\frac{(1-\eta)}{2}\left\{\frac{\beta_{W} \zeta_{3} \varepsilon_{W}}{\tau_{W} \rho_{E H}}\right. \\
& +\left[\beta _ { W } \left(\frac{4\left(\rho_{E H}+\varepsilon_{W} \zeta_{1}\right)\left(\sigma_{C H}+k_{2} \eta_{1}\right) \beta_{C}}{\rho_{E H} \tau_{W}\left(k_{1} k_{2}-\sigma_{C H} \gamma_{C H}\right)} \frac{U_{P}^{*}}{U_{W}^{*}}\right.\right. \\
& \frac{4\left(\rho_{E H}+\varepsilon_{W} \zeta_{2}\right)\left(k_{4} \eta_{1}+\sigma_{H}\right) \beta_{H}}{\rho_{E H} \tau_{W}\left(k_{3} k_{4}-\sigma_{H} \gamma_{H}\right)} \frac{U_{P}^{*}}{U_{W}^{*}} \\
& \left.\left.\left.+\frac{\zeta_{3}^{2} \varepsilon_{W}^{2} \beta_{W}}{\tau_{W}^{2} \rho_{E H}^{2}}\right)^{1 / 2}\right]\right\} .
\end{aligned}
$$

The result below is a consequence of Theorem 2 of [48].

Lemma 2. The disease free equilibrium (DFE) of the hospital setting is locally asymptotically stable (LAS) when $\mathcal{R}_{H C}<1$, and unstable when $\mathcal{R}_{H C}>1$.

The quantity $\mathcal{R}_{H C}$ represents the reproduction number of individuals with MRSA in the hospital. The threshold quantity $\mathcal{R}_{H C}$ measures the average number of new infections generated by a single infected individual in a completely-susceptible population [2], [15], [24], [48]. Thus, Lemma 2 implies that the MRSA can be eliminated (when $\mathcal{R}_{H C}<1$ ) if the initial sizes of the sub-populations of the model (2.1) are in the basin of attraction of the DFE $\left(\mathcal{E}_{H C}\right)$.

\section{Interpretation of $\mathcal{R}_{H C}$}

The basic reproduction number, given by Equation (1), can be re-written in the following convenient form: 


$$
\begin{aligned}
& \mathcal{R}_{H C}=\frac{(1-\eta)}{2}\left\{\frac{\beta_{W} \zeta_{3} \varepsilon_{W}}{\rho_{E H} \tau_{W}}\right. \\
& +\left[\beta _ { W } \left(\frac{4\left(\sigma_{C H}+k_{2} \eta_{1}\right)\left(\rho_{E H}+\varepsilon_{W} \zeta_{1}\right) \beta_{C}}{\left(k_{1} k_{2}-\sigma_{C H} \gamma_{C H}\right) \tau_{W} \rho_{E H}} \frac{U_{P}^{*}}{U_{W}^{*}}\right.\right. \\
& \frac{4\left(k_{4} \eta_{1}+\sigma_{H}\right)\left(\rho_{E H}+\varepsilon_{W} \zeta_{2}\right) \beta_{H}}{\left(k_{3} k_{4}-\sigma_{H} \gamma_{H}\right) \rho_{E H} \tau_{W}} \frac{U_{P}^{*}}{U_{W}^{*}} \\
& \left.\left.\left.+\frac{\zeta_{3}^{2} \varepsilon_{W}^{2} \beta_{W}}{\tau_{W}^{2} \rho_{E H}^{2}}\right)^{1 / 2}\right]\right\} .
\end{aligned}
$$

The epidemiological quantity, $\mathcal{R}_{H C}$, can be interpreted as follows. The first term in 3.2 measures the average number of new cases generated due to the contaminated health-care workers $\left(E_{H}\right)$. It is the product of the contamination rate of uncontaminated environment $E_{H}$ by the contaminated health-care workers $C_{W}\left(\frac{\beta_{W} \zeta_{3} U_{W}^{*}}{N_{W}^{*}}=\beta_{W} \zeta_{3} ;\right.$ since $\left.N_{W}^{*}=U_{W}^{*}\right)$, the probability of been in the $E_{H}$ class $\left(\frac{\varepsilon_{W}}{\rho_{E H}}\right)$ and the average duration in the $C_{W}$ class $\left(\frac{1}{\tau_{W}}\right)$.

The square root term in 3.2 accounts for the average number of new infections generated by patients with CA-MRSA, HA-MRSA and the contaminated environment due to their contact with contaminated health-care workers. The first term in the square root is the contribution to the average number of new infections due to the interactions of the health-care workers and patients with CAMRSA. The second term in the square root gives the average number of new infections generated by patients with HA-MRSA due to their interactions with health-care workers. The third term gives the average number of new infections generated by the environment due to their contact with health-care workers. The sum of these terms gives the basic reproduction number, $\mathcal{R}_{H C}$. The disease can be effectively controlled if $\mathcal{R}_{H C}$ is less than unity, and will persist if it exceeds unity.

In the next section, an analogous stochastic model for model (2.1) is formulated using a continuous time Markov chain.

\section{The Stochastic MOdEL OF CA- AND HA- MRSA MODEL}

In this section we model the system as a stochastic process. For any time $t \geq 0$, let $\mathcal{U}_{\mathcal{P}}(t)$, $\mathcal{C}_{\mathcal{C H}}(t), \quad \mathcal{I}_{\mathcal{C H}}(t), \quad \mathcal{C}_{\mathcal{H}}(t), \quad \mathcal{I}_{\mathcal{H}}(t), \quad \mathcal{Q}(t), \quad \mathcal{U}_{\mathcal{W}}(t)$, $\mathcal{C}_{\mathcal{W}}(t)$, and $\mathcal{E}_{\mathcal{H}}(t)$ be discrete random variables denoting the numbers of uncolonized patients, patients colonized with CA-MRSA strain, patients infected with CA-MRSA, patients colonized with HA-MRSA strain, patients infected with HAMRSA, isolated patients, uncontaminated healthcare workers, contaminated health-care workers, and amount of colonization in the overall hospital environment, respectively. The process

$$
\begin{array}{r}
\left\{\left(\mathcal{U}_{\mathcal{P}}(t), \mathcal{C}_{\mathcal{C H}}(t), \mathcal{I}_{\mathcal{C H}}(t), \mathcal{C}_{\mathcal{H}}(t), \mathcal{I}_{\mathcal{H}}(t), \mathcal{Q}(t),\right.\right. \\
\left.\left.\mathcal{U}_{\mathcal{W}}(t), \mathcal{C}_{\mathcal{W}}(t), \mathcal{E}_{\mathcal{H}}(t)\right), \quad t \geq 0\right\}
\end{array}
$$

evolves over time as a vector-valued, timehomogeneous, continuous-time Markov chain (CTMC) over the state space $\Omega:=\left\{\left(x_{1}, \ldots, x_{9}\right)\right\}$ with $x_{8}+x_{9}=K_{W}$, a constant, and $x_{i} \in$ $\{0,1,2, \ldots\}$ otherwise. Here $K_{W}$ is the total (constant) number of health-care workers. The other processes are bounded below by zero, but unconstrained otherwise, assuming the health-care facility effectively has no capacity constraints limiting the number of patients or the environmental colonization level. We will use lower-case letters

$$
\left\{u_{p}, c_{c h}, i_{c h}, c_{h}, i_{h}, q, u_{w}, c_{w}, e_{h}\right\}
$$

as well as $x_{1}, \ldots, x_{9}$ to denote the values of the respective processes. Let

$$
q_{x y}=\lim _{h \rightarrow 0} h^{-1} P(X(h)=y \mid X(0)=x)
$$

denote the instantaneous transition rates of the process, meaning the rate at which the process attempts a jump from state $x$ into state $y$ given it is currently in state $x$. Also for $i \in\{1, \ldots, 9\}$ let $e_{i}$ denote the unit vector with a 1 in the $i$ th position. By examining the flow diagram in Figure 1, for any state $x$, the only allowed transitions for the vector-valued process in (1) are among the types 
$x \rightarrow x+e_{i}, x \rightarrow x-e_{i}$, or $x \rightarrow x-e_{i}+e_{j}$, denoting a movement from outside the system into compartment $i$, a movement from compartment $i$ to outside the system, and a movement from compartment $i$ to compartment $j$, respectively. Then the infinitesimal transition rates for our process are given by

$$
\begin{aligned}
& q_{x, x+e_{u_{p}}}=\Pi_{P}\left(1-\pi_{C C}-\pi_{I C}-\pi_{C H}-\pi_{I H}\right) \\
& q_{x, x-e_{u_{p}}}=\left(\mu_{H}+\alpha_{P}\right) u_{p} \\
& q_{x, x+e_{c_{c h}}}=\pi_{C C} \Pi_{P} \\
& q_{x, x-e_{u_{p}}+e_{c_{c h}}}=\left[\frac{\beta_{C}\left(c_{w}+\zeta_{1} e_{h}\right)(1-\eta)}{n_{w}}\right] u_{p} \\
& q_{x, x-e_{c_{c h}}+e_{u_{p}}}=\tau_{C H} c_{c h} \\
& q_{x, x-e_{c_{c h}}+e_{i_{c h}}}=\sigma_{C H} c_{c h} \\
& q_{x, x-e_{c_{c h}}+e_{q}}=\omega_{C C H} c_{c h} \\
& q_{x, x-e_{c_{c h}}}=\left(\mu_{H}+\alpha_{C}\right) c_{c h} \\
& q_{x, x+e_{i_{c h}}}=\pi_{I C} \Pi_{P}
\end{aligned}
$$

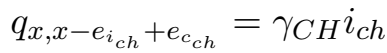

$$
\begin{aligned}
& q_{x, x-e_{i_{c h}}+e_{q}}=\omega_{I C H} i_{c h} \\
& q_{x, x-e_{i_{c h}}}=\left(\mu_{H}+\delta_{C H}\right) i_{c h} \\
& q_{x, x+e_{c_{h}}}=\pi_{C H} \Pi_{P} \\
& q_{x, x-e_{u_{p}}+e_{c_{h}}}=\left[\frac{\beta_{H}\left(c_{w}+\zeta_{2} e_{h}\right)(1-\eta)}{n_{w}}\right] u_{p} \\
& q_{x, x-e_{c_{h}}+e_{u_{p}}}=\tau_{H} c_{h} \\
& q_{x, x-e_{c_{h}}+e_{i_{h}}}=\sigma_{H} c_{h} \\
& q_{x, x-e_{c_{h}}+e_{q}}=\omega_{C H} c_{h} \\
& q_{x, x-e_{c_{h}}}=\left(\mu_{H}+\alpha_{H}\right) c_{h} \\
& q_{x, x+e_{i_{h}}}=\pi_{I H} \Pi_{P} \\
& q_{x, x-e_{i_{h}}+e_{c_{h}}}=\gamma_{H} i_{h} \\
& q_{x, x-e_{i_{h}}+e_{q}}=\omega_{I H} i_{h} \\
& q_{x, x-e_{i_{h}}}=\left(\mu_{H}+\delta_{H}\right) i_{h} \\
& q_{x, x-e_{q}}=\left(\mu_{H}+\delta_{Q}+\alpha_{Q}\right) q \\
& q_{x, x-e_{u_{w}}+e_{c_{w}}}=u_{w}\left(\beta _ { W } \left[\eta_{1}\left(c_{c h}+c_{h}\right)+i_{c h}\right.\right. \\
& \left.\left.+i_{h}+\zeta_{3} e_{h}\right](1-\eta)\right) /\left(n_{w}\right) \\
& q_{x, x-e_{c_{w}}+e_{u_{w}}}=\tau_{W} c_{w} \\
& q_{x, x+e_{e_{h}}}=\varepsilon_{W} c_{w} \\
& q_{x, x-e_{e_{h}}}=\rho_{E H} e_{h}
\end{aligned}
$$

and 0 otherwise. Note our model does not allow for permanent extinction of either bacterium due to the non-zero arrival rates of colonized and infected patients from outside the system.

Many disease transmission models such as those in [6], [29], and [30] assume fixed population sizes. Although the patient capacity of our hospital is unconstrained from above, the form of the state transition rates ensures the process is positive recurrent, meaning if the process begins in an arbitrary state $x$ at time 0 , the process returns to state $x$ in a finite amount of time with probability 1 and the expected time to do so is finite.

Proposition 1. The process in (1) is positive recurrent.

\section{Sensitivity Analysis}

Deterministic model output is determined by its input parameters, which exhibit some uncertainty in the process of their selection. To assess the impact of uncertainty and sensitivity of the outcomes of the numerical simulation of the model (2.1) to variations in each parameter, a global sensitivity analysis was carried out using Latin Hypercube Sampling (LHS) and partial rank correlation coefficients (PRCC). LHS is a stratified sampling without replacement technique which allows for an efficient analysis of parameter variations across simultaneous uncertainty ranges in each parameter [3], [32], [33], [40]. PRCC measures the strength of the relationship between the model outcome and the parameters, stating the degree of the effect that each parameter has on the outcome [3], [32], [33], [40]. A total of 1,000 simulations of the model (2.1) per LHS run were carried out, using the parameters values given in Table II (with the stated ranges).

The aim in this section is to determine the parameters with the largest impact on the model outcome. The reproduction number $\mathcal{R}_{H C}$, given by (1), was used as the model outcome. The PRCC for each parameter used in the sensitivity analysis is depicted in Figure 2(a) (and also tabulated in Table III). It follows from Figure 2(a) and Table 
III that the key parameters that influence the value of $\mathcal{R}_{H C}$ are the environmental contamination rate $\left(\varepsilon_{W}\right)$, the bacterial decay rate $\left(\rho_{E H}\right)$, hand washing compliance rate $(\eta)$, the health-care workers' decontamination rate $\left(\tau_{W}\right)$ and the transmission probability of health-care workers per contact with patients $\left(\beta_{W}\right)$.

The sensitivity analysis was also carried out using prevalence of CA-MRSA among patients as output (and the results obtained are given in Figure 2(b), and Table [III). The dominant parameters in this case are the transmission probability of CA-MRSA per contact with health-care workers $\left(\beta_{C}\right)$, hand washing compliance rate $(\eta)$, environmental contamination rate $\left(\varepsilon_{W}\right)$, the transmission probability of HA-MRSA per contact with healthcare workers $\left(\beta_{H}\right)$, isolation rate of patients with CA-MRSA $\left(\omega_{C C H}\right)$, the admission rates into the hospital $\left(\Pi_{P}\right)$.

The sensitivity analysis was also carried out using prevalence of HA-MRSA among patients as output (and the results obtained are given in Figure 2(c), and Table III). The dominant parameters in this case are the transmission probability of CAMRSA per contact with health-care workers $\left(\beta_{C}\right)$, hand washing compliance rate $(\eta)$, environmental contamination rate $\left(\varepsilon_{W}\right)$, the transmission probability of CA-MRSA per contact with health-care workers $\left(\beta_{H}\right)$, the admission rates into the hospital $\left(\Pi_{P}\right)$, and isolation rate of patients with HA$\operatorname{MRSA}\left(\omega_{C H}\right)$.

The sensitivity analysis was also carried out using as output the prevalence of MRSA among health-care workers (and the results obtained are given in Figure 2(d) and Table (III). The dominant parameters here are the admission rates into the hospital $\left(\Pi_{P}\right)$, the transmission probability of CA-MRSA per contact with health-care workers $\left(\beta_{C}\right)$, the health-care workers decontamination rate $\left(\tau_{W}\right)$, isolation rate of patients with HA-MRSA $\left(\omega_{C H}\right)$, the transmission probability of health-care workers per contact with patients $\left(\beta_{W}\right)$, and the transmission probability of HA-MRSA per contact with health-care workers $\left(\beta_{H}\right)$.

To further analyze the parameters which have the dominant impact, we used the hospital environment as model output (and the results are given in Figure 2(e) and Table III). The dominant parameters here are environmental contamination rate $\left(\varepsilon_{W}\right)$, hand washing compliance rate $(\eta)$, the admission rates into the hospital $\left(\Pi_{P}\right)$, the transmission probability of health-care workers per contact with patients $\left(\beta_{W}\right)$, the environmental contamination rate $\left(\varepsilon_{W}\right)$, the transmission probability of CA-MRSA per contact with health-care workers $\left(\beta_{C}\right)$, and the transmission probability of HA-MRSA per contact with health-care workers $\left(\beta_{H}\right)$.

For each of the 1,000 runs, the prevalence of CA-MRSA among patients is generated as the output. Figure 3(a) display the boxplots of the total number of colonized patients, for $t=$ $20,40, \cdots, 100$ days. It follows from this figure that the prevalence of CA-MRSA among patients increase monotonically over $t=20,40, \cdots, 100$. The presence of outliers in this figure indicates that certain parameter configurations result in values of the prevalence of CA-MRSA among patients that are markedly higher than the typical values inside the boxes. Similar observations are seen in Figures 3(b) and 3(d) for the boxplots of prevalence of HAMRSA among patients and prevalence of MRSA (CA-MRSA and HA-MRSA) among health-care workers and hospital environment.

Comparing Figures 3(a) and 3(b), we observed at every time point an increase in the difference in the prevalence of CA-MRSA and HA-MRSA in the hospital, reaching about $10 \%$ at the end of our simulation period. Figure 3(c) on the other hand, shows that MRSA prevalence among the healthcare workers is higher at every time point than the prevalence among the hospital patience.

Hence, the aforementioned sensitivity analysis of the model 2.1 suggests that to curtail the trans- 


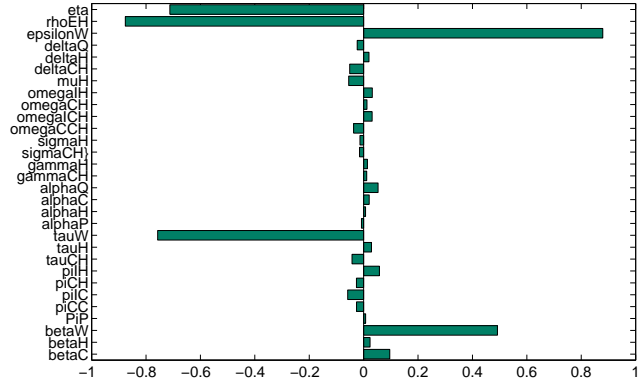

(a)

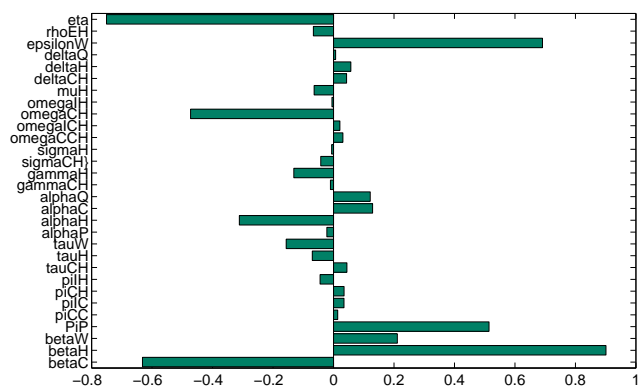

(c)

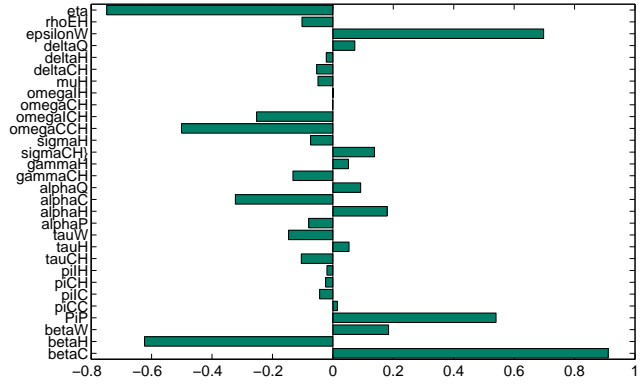

(b)

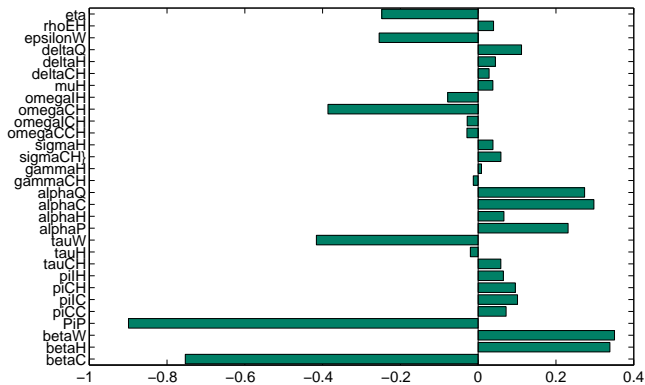

(d)

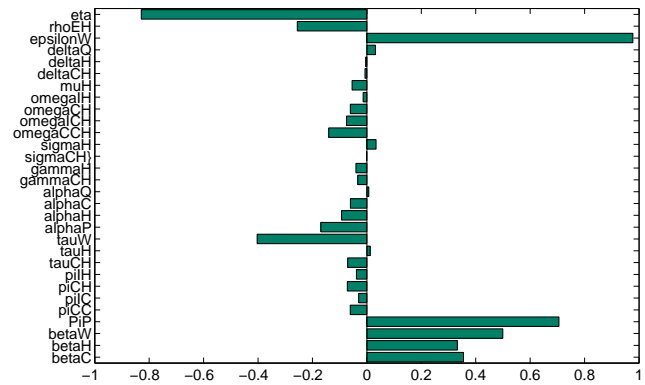

(e)

Fig. 2. PRCC for the model (2.1), using as output (a) $\mathcal{R}_{H C}$; (b) prevalence of CA-MRSA among patients; (c) prevalence of HA-MRSA among patients; (d) prevalence MRSA among health-care workers; (e) the hospital environment. 
F. B. Agusto et al., Modeling of Community and Hospital-acquired Methicillin-resistant ...

\begin{tabular}{|c|c|c|c|}
\hline Parameter & Baseline values & Ranges & Reference \\
\hline$\beta_{C}$ & 0.223 & $(0.045-0.4)$ & [11], [39] \\
\hline$\beta_{H}$ & 0.224 & $(0.048-0.4)$ & [11], [39] \\
\hline$\beta_{W}$ & 0.023 & $(0.015-0.03)$ & [6], |21] \\
\hline$\eta$ & 0.62 & $(0.40-0.83)$ & [11], [39], [19], [51] \\
\hline$\eta_{1}, \zeta_{1}, \zeta_{2}, \zeta_{3}$ & variable & variable & \\
\hline$\Pi_{P}$ & 230 & $(50-400)$ & 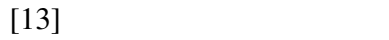 \\
\hline$\pi_{C C}$ & 0.03 & $(0.00063-0.057)$ & [9], [13], [39] \\
\hline$\pi_{I C}$ & 0.07 & $"$ & [13] \\
\hline$\pi_{C H}$ & 0.005 & $"$ & [13], [39] \\
\hline$\pi_{I H}$ & 0.0017 & $"$ & [13] \\
\hline$\tau_{C H}$ & 0.0021 & $\left(1 / 370-0.10 \times \frac{1}{7}\right)$ & [6], 9] \\
\hline$\tau_{H}$ & 0.004 & $"$ & $"$ \\
\hline$\tau_{W}$ & 0.003 & $"$ & $"$ \\
\hline$\mu_{H}$ & 0.008 & $(0.0001-0.001)$ & Assumed \\
\hline$\alpha_{P}$ & $\frac{1}{5}$ & $\left(\frac{1}{8}-\frac{1}{5}\right)$ & [6], [9], [13], [17], [39], [21] \\
\hline$\alpha_{H}$ & $\frac{1}{7}$ & & [6], [17], [39] \\
\hline$\alpha_{C}$ & $\frac{1}{8}$ & $"$ & $"$ \\
\hline$\alpha_{Q}$ & $\frac{1}{5}$ & $"$ & $"$ \\
\hline$\gamma_{C H}$ & $0.967 \times \frac{1}{10}$ & $(0.08-0.025)$ & [13] \\
\hline$\gamma_{H}$ & 0.0436 & $\left(0.6 \times \frac{1}{14}-0.8 \times \frac{1}{18}\right)$ & 6], [13 \\
\hline$\sigma_{C H}$ & $0.10 \times \frac{1}{5}$ & $\left(0.10 \times \frac{1}{7}-0.30 \times \frac{1}{7}\right)$ & [13] \\
\hline$\sigma_{H}$ & $0.10 \times \frac{1}{5}$ & & [6], 13] \\
\hline$\omega_{C C H}$ & 0.06 & $(0.02-0.1)$ & [9], [44] \\
\hline$\omega_{I C H}$ & 0.065 & $"$ & $"$ \\
\hline$\omega_{C H}$ & 0.063 & ” & ” \\
\hline$\omega_{I H}$ & 0.065 & $"$ & $"$ \\
\hline$\delta_{C H}$ & $\frac{1}{10} \times 0.033$ & $\left(0.20 \times \frac{1}{18}-0.4 \times \frac{1}{14}\right)$ & [13] \\
\hline$\delta_{H}$ & $\frac{1}{10} \times 0.20$ & & [6], [13] \\
\hline$\delta_{Q}$ & 0.0198 & $"$ & Assumed \\
\hline$\varepsilon_{W}$ & 0.5 & $(0.04-1.0)$ & [18], [22], 25] \\
\hline$\rho_{E H}$ & 0.001 & $(0.0002-0.00353)$ & [10], [20], [31] \\
\hline
\end{tabular}

TABLE II

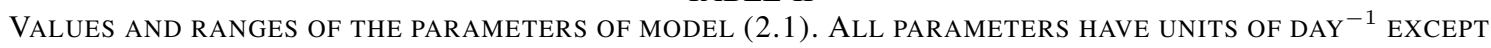
$\beta_{C}, \beta_{H}, \beta_{W}, \eta_{1}, \zeta_{1}, \zeta_{2}, \zeta_{3}, \pi_{C C}, \pi_{I C}, \pi_{C H}, \pi_{I H}$, WHICH ARE UNITLESS PROPORTIONS, AND THE ARRIVAL RATE $\Pi_{P}$ HAS UNITS OF NUMBER PER DAY.

mission of MRSA in the hospital control efforts should be focused on increasing the hand washing compliance rate (by increasing $\eta$ ), increasing the health-care workers decontamination rate (by increasing $\tau_{W}$ ), reducing the environmental contamination rate (by decreasing $\varepsilon_{W}$ ), ensuring that the admission rates into the hospital $\left(\Pi_{P}\right)$ does not exceed the number of available beds and increasing the isolation rate of patients with CA-MRSA and HA-MRSA (by increasing $\omega_{C C H}$ and $\omega_{C H}$ rates). The analysis further suggests that MRSA transmission can curtail by reducing the transmission of CA- and HA-MRSA to health- care workers (via the reduction of $\beta_{C}$ and $\beta_{H}$ ) and reducing the transmission from health-care workers to the patients (via the reduction of $\beta_{W}$ ).

In summary, sensitivity analysis of the model 2.1 suggests the following:

(a) the key parameters that influences the value of $\mathcal{R}_{H C}$ are the environmental contamination rate $\left(\varepsilon_{W}\right)$, the bacterial decay rate $\left(\rho_{E H}\right)$, hand washing compliance rate $(\eta)$, the health-care workers decontamination rate $\left(\tau_{W}\right)$ and the transmission probability of health-care workers per contact with patients 
F. B. Agusto et al., Modeling of Community and Hospital-acquired Methicillin-resistant ...

\begin{tabular}{|c|c|c|c|c|c|}
\hline Parameters & $\begin{array}{l}\text { PRCC } \\
\mathcal{R}_{H C}\end{array}$ & $\begin{array}{l}\text { PRCC } \\
\text { Prevalence } \\
\text { of CA-MRSA } \\
\text { among patients } \\
\end{array}$ & $\begin{array}{l}\text { PRCC } \\
\text { Prevalence } \\
\text { of HA-MRSA } \\
\text { among patients }\end{array}$ & $\begin{array}{l}\text { PRCC } \\
\text { Prevalence of MRSA } \\
\text { among Health-care } \\
\text { workers }\end{array}$ & $\begin{array}{l}\text { PRCC } \\
\text { Environment }\end{array}$ \\
\hline$\beta_{C}$ & 0.0960 & 0.9115 & -0.6316 & -0.7531 & 0.3550 \\
\hline$\beta_{H}$ & 0.0236 & -0.6234 & 0.9011 & 0.3388 & 0.3323 \\
\hline$\beta_{W}$ & 0.4921 & 0.1843 & 0.2112 & 0.3506 & 0.4993 \\
\hline$\Pi_{P}$ & 0.0074 & 0.5402 & 0.5144 & -0.8991 & 0.7058 \\
\hline$\pi_{C C}$ & -0.0262 & 0.0149 & 0.0141 & 0.0719 & -0.0611 \\
\hline$\pi_{I C}$ & -0.0587 & -0.0438 & 0.0347 & 0.1010 & -0.0300 \\
\hline$\pi_{C H}$ & -0.0264 & -0.0240 & 0.0349 & 0.0955 & -0.0720 \\
\hline$\pi_{I H}$ & 0.0580 & -0.0197 & -0.0444 & 0.0647 & -0.0386 \\
\hline$\tau_{C H}$ & -0.0426 & -0.1046 & 0.0445 & 0.0582 & -0.0705 \\
\hline$\tau_{H}$ & 0.0290 & 0.0535 & -0.0700 & -0.0201 & 0.0121 \\
\hline$\tau_{W}$ & -0.7572 & -0.1467 & -0.1563 & -0.4161 & -0.4031 \\
\hline$\alpha_{P}$ & -0.0079 & -0.0799 & -0.0220 & 0.2312 & -0.1689 \\
\hline$\alpha_{H}$ & 0.0070 & 0.1797 & -0.3113 & 0.0663 & -0.0931 \\
\hline$\alpha_{C}$ & 0.0205 & -0.3226 & 0.1292 & 0.2975 & -0.0605 \\
\hline$\alpha_{Q}$ & 0.0531 & 0.0922 & 0.1216 & 0.2738 & 0.0070 \\
\hline$\gamma_{C H}$ & 0.0113 & -0.1323 & -0.0102 & -0.0126 & -0.0331 \\
\hline$\gamma_{H}$ & 0.0138 & 0.0516 & -0.1313 & 0.0086 & -0.0404 \\
\hline$\sigma_{C H}$ & -0.0151 & 0.1376 & -0.0423 & 0.0585 & -0.0013 \\
\hline$\sigma_{H}$ & -0.0136 & -0.0736 & -0.0061 & 0.0380 & 0.0339 \\
\hline$\omega_{C C H}$ & -0.0370 & -0.5009 & 0.0312 & -0.0289 & -0.1401 \\
\hline$\omega_{I C H}$ & 0.0312 & -0.2523 & 0.0210 & -0.0284 & -0.0748 \\
\hline$\omega_{C H}$ & 0.0123 & -0.0008 & -0.4727 & -0.3862 & -0.0608 \\
\hline$\omega_{I H}$ & 0.0319 & 0.0010 & -0.0052 & -0.0783 & -0.0139 \\
\hline$\mu_{H}$ & -0.0550 & -0.0492 & -0.0640 & 0.0378 & -0.0544 \\
\hline$\delta_{C H}$ & -0.0512 & -0.0536 & 0.0434 & 0.0282 & -0.0069 \\
\hline$\delta_{H}$ & 0.0198 & -0.0219 & 0.0574 & 0.0442 & -0.0041 \\
\hline$\delta_{Q}$ & -0.0235 & 0.0727 & 0.0070 & 0.1115 & 0.0314 \\
\hline$\epsilon_{W}$ & 0.8793 & 0.6976 & 0.6913 & -0.2547 & 0.9773 \\
\hline$\rho_{E H}$ & -0.8760 & -0.1021 & -0.0666 & 0.0395 & -0.2557 \\
\hline$\eta$ & -0.7125 & -0.7483 & -0.7506 & -0.2478 & -0.8289 \\
\hline
\end{tabular}

TABLE III

PRCC FOR MODEL (2.1) USING AS OUTPUT $\mathcal{R}_{H C}$, PREVALENCE OF CA-MRSA, PREVALENCE OF HA-MRSA, PREVALENCE AMONG HEALTH-CARE WORKERS AND THE HOSPITAL ENVIRONMENT.

$\left(\beta_{W}\right)$

(b) the dominant parameters impacting the prevalence of CA-MRSA among patients are the transmission probability of CA-MRSA per contact with health-care workers $\left(\beta_{C}\right)$, hand washing compliance rate $(\eta)$, environmental contamination rate $\left(\varepsilon_{W}\right)$, the transmission probability of HA-MRSA per contact with health-care workers $\left(\beta_{H}\right)$, isolation rate of patients with CA-MRSA $\left(\omega_{C C H}\right)$, the admission rates into the hospital $\left(\Pi_{P}\right)$.

(c) the dominant parameters influencing the prevalence of HA-MRSA among patients are the transmission probability of HAMRSA per contact with health-care workers $\left(\beta_{C}\right)$, hand washing compliance rate $(\eta)$, environmental contamination rate $\left(\varepsilon_{W}\right)$, the transmission probability of CA-MRSA per contact with health-care workers $\left(\beta_{H}\right)$, the admission rates into the hospital $\left(\Pi_{P}\right)$, isolation rate of patients with HA-MRSA $\left(\omega_{C H}\right)$.

(d) the key parameters impacting the prevalence of MRSA among health-care workers are the admission rates into the hospital $\left(\Pi_{P}\right)$, the transmission probability of CA-MRSA per contact with health-care workers $\left(\beta_{C}\right)$, the health-care workers decolonization rate $\left(\tau_{W}\right)$, isolation rate of patients with HAMRSA $\left(\omega_{C H}\right)$, the transmission probability of health-care workers per contact with patients $\left(\beta_{W}\right)$, the transmission probability of HA-MRSA per contact with health-care workers $\left(\beta_{H}\right)$.

(e) the key parameters influencing the hospital environment are environmental contamination rate $\left(\varepsilon_{W}\right)$, hand washing compliance rate $(\eta)$, the admission rates into the hospital $\left(\Pi_{P}\right)$, the transmission probability of healthcare workers per contact with patients $\left(\beta_{W}\right)$, the environmental contamination rate $\left(\varepsilon_{W}\right)$, the transmission probability of CA-MRSA per contact with health-care workers $\left(\beta_{C}\right)$, the transmission probability of HA-MRSA per contact with health-care workers $\left(\beta_{H}\right)$. 
F. B. Agusto et al., Modeling of Community and Hospital-acquired Methicillin-resistant ...

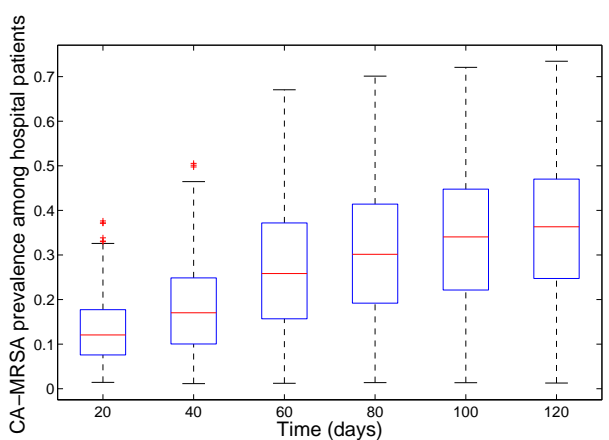

(a)

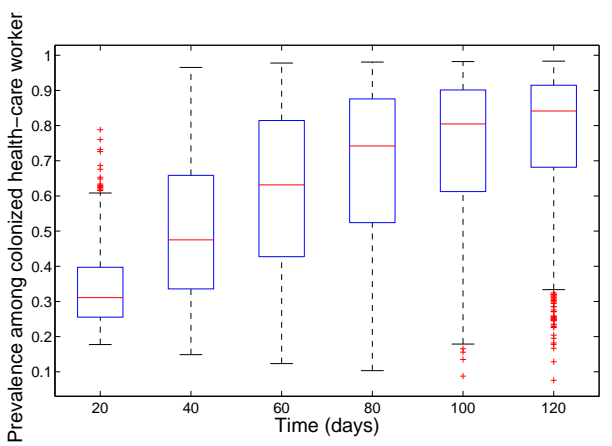

(c)

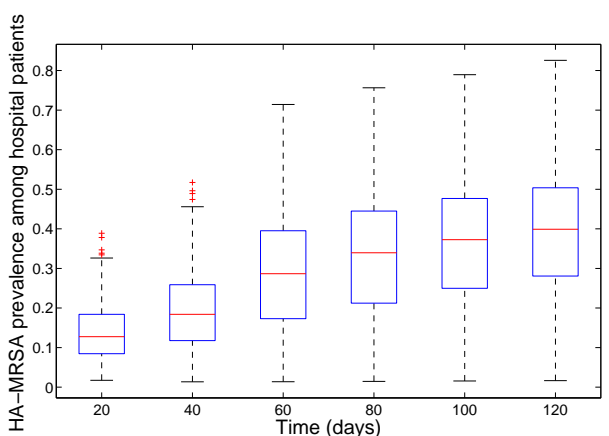

(b)

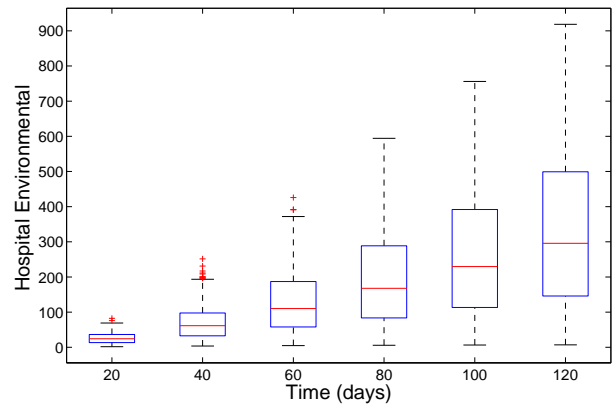

(d)

Fig. 3. (a). Boxplots of prevalence of CA-MRSA among patients; (b). Boxplots of prevalence of HA-MRSA among patients; (c). Boxplots of prevalence of MRSA among health-care workers; (d). Boxplots of the hospital environment.

\section{Vi. Assessment of Basic Control MEASURES}

In order to reduce the number of MRSA cases in the hospital the different dominant parameters values were adjusted following the results of the sensitivity analysis in Section $\mathrm{V}$ above. This was done to reflect three control strategies: low-effectiveness strategy, moderate-effectiveness strategy, and high-effectiveness strategy.

\section{A. Low-effectiveness control strategy}

The low-effectiveness MRSA control strategy assumes the health-care workers hand washing compliance rate to be about $40 \%$ (i.e., $\eta=0.40$ ) and the health-care workers do not decontaminate often (so that $\tau_{W}=1 / 370$ ). As a result there is a high level of bacteria in the environmental, leading to a high contamination rate (hence, $\varepsilon_{W}=1.0$ ). We assume that the hospital admission rates is at the maximum capacity (i.e $\Pi_{P}=400$ ). For this control strategy we set the isolation rate of patients with CA-MRSA and HA-MRSA as $\left(\omega_{C C H}=0.02\right)$ and $\left(\omega_{C H}=0.02\right)$. In similar manner, we set the transmission probabilities of CA- and HA-MRSA per contact with health-care workers (i.e., $\beta_{C}=0.4$ and $\beta_{H}=0.4$ ) and transmission probability of health-care workers per contact with patients (i.e., $\beta_{W}=0.03$ ).

\section{B. Moderate-effectiveness control strategy}

For the moderate-effectiveness level of the antiMRSA control strategy, the health-care workers hand washing compliance rate is increased to about $60 \%$ (i.e., $\eta=0.60$ ) and the health-care workers decontamination rate is also increased (so that $\tau_{W}=0.003$ ). As a result there is a reduction is a reduction in the level of bacteria in the environmental, leading to a lower contamination 
rate (hence, $\varepsilon_{W}=0.5$ ). We reduce the hospital number admitted (i.e $\Pi_{P}=230$ ). We increase the isolation rate of patients with CA-MRSA and HAMRSA as $\left(\omega_{C C H}=0.06\right)$ and $\left(\omega_{C H}=0.063\right)$. In similar manner, we reduce the transmission probabilities of CA- and HA-MRSA per contact with health-care workers (i.e., $\beta_{C}=0.223$ and $\left.\beta_{H}=0.224\right)$ and transmission probability of health-care workers per contact with patients (i.e., $\left.\beta_{W}=0.023\right)$.

\section{High-effectiveness control strategy}

For the high-effectiveness level of the MRSA control strategy, the health-care workers hand washing compliance rate is increased to $83 \%$ and also increase the health-care workers decontamination rate (so that $\tau_{W}=0.10 \times \frac{1}{7}$ ). The environmental contamination rate is reduced (hence, $\varepsilon_{W}=0.04$ ). We reduce the hospital number admitted (i.e $\Pi_{P}=130$ ). We increase the isolation rate of patients with CA-MRSA and HA-MRSA as $\left(\omega_{C C H}=0.1\right)$ and $\left(\omega_{C H}=0.1\right)$. In similar manner, we reduce the transmission probabilities of CA- and HA-MRSA per contact with health-care workers (i.e., $\beta_{C}=0.045$ and $\left.\beta_{H}=0.048\right)$ and transmission probability of health-care workers per contact with patients (i.e., $\left.\beta_{W}=0.015\right)$.

Figure 4(a) depicts the prevalence of community acquired MRSA among hospital patients under the various control strategies over a 60-day period, from which it can be observed that about $80 \%$ cases were obtained with the low-effective strategy and about $60 \%$ for the moderate-effective strategy. However, for the high-effectiveness control strategy, we observed a gradual decrease and eventual elimination of MRSA in the hospital. We observed similar behavior in Figure 4(b) for the prevalence of hospital acquired MRSA among the hospital patients under these control strategies. On the other hand Figure 4(c), shows that with the low-effective strategy the prevalence of MRSA among the health-care workers quickly reaches $100 \%$; however with the moderate-effective strategy the prevalence of MRSA slowly reaches
$100 \%$. Figure 4(c), also depict the prevalence of MRSA among the health-care workers with the high-effectiveness control strategy, which unlike the other two strategies reduces quickly prevalence of MRSA among the health-care workers and eventually eliminating it the hospital. As expected, the high-effectiveness of the control strategy is far more effective in curtailing the prevalence of MRSA in the hospital. It is worth noting, however, that the moderate-effectiveness strategy also resulted in a reduction on the prevalence of MRSA among hospital patients in comparison to the low-effectiveness strategy. However, this is not the case among the health-care workers. The high-effectiveness of the control strategy is the best strategy to implement with the healthcare workers. These simulations clearly show that MRSA are controllable using basic health control measures, such as the moderate- and high-effectiveness levels of the control strategy described above.

\section{Mean time to colonization}

Next we investigate for he deterministic model (2.1) the mean time to colonization, while still using the control strategies above; this is defined as the average time a susceptible patient remain susceptible upon admission into the hospital [6] and it is give as:

$$
W(t)=\frac{1}{\left[\lambda_{C H}(t)+\lambda_{H}(t)+\alpha_{P}+\mu_{H}\right] U_{P}(t)} .
$$

We observe from Figure 5 an increase in the mean time to colonization with increasing effectiveness level. In other words, the high-effectiveness strategy leads to the lengthiest mean time to colonization, this is followed by the moderate-effectiveness strategy and then the low-effective strategy which produces the least mean time to colonization. This result indicate that the high-effective strategy has the slowest colonization rate while with the loweffective strategy patients are colonized quickly in the hospital. 
F. B. Agusto et al., Modeling of Community and Hospital-acquired Methicillin-resistant ...

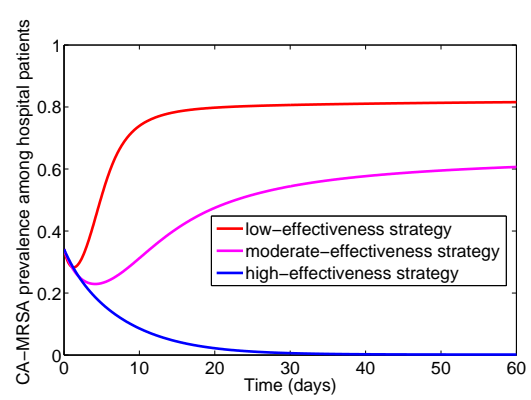

(a)

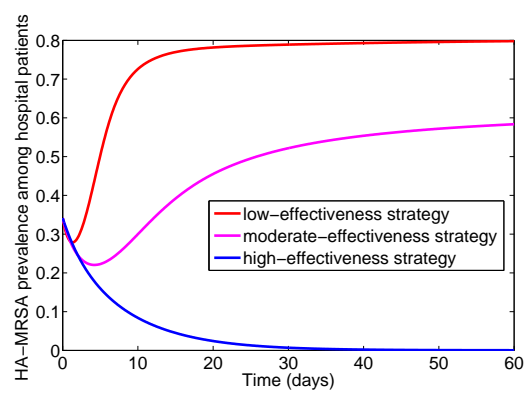

(b)

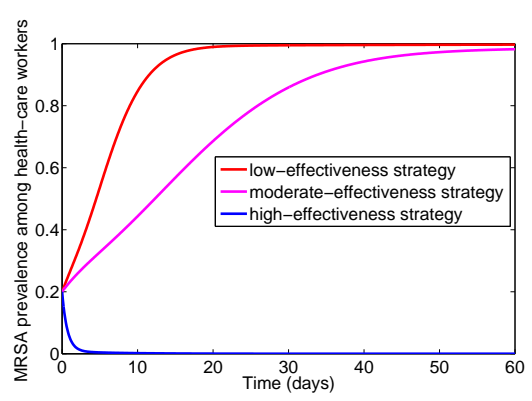

(c)

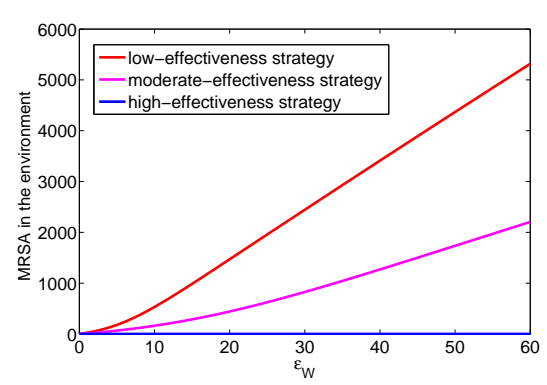

(d)

Fig. 4. Simulations of the model 2.1, showing the prevalence cases generated under various effectiveness levels of the control strategies. (a). CA-MRSA prevalence among hospital patients. (b). HA-MRSA prevalence among hospital patients. (c). MRSA prevalence among health-care workers. (d). MRSA in the environment. Other parameter values used are as given in Table II

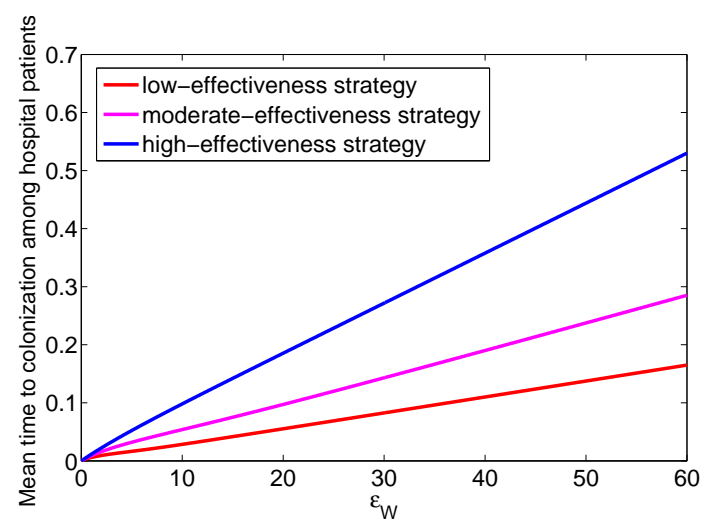

Fig. 5. Simulations of the model 2.1, showing the Mean time to colonization among hospital patients under various effectiveness levels of the control strategies. Other parameter values used are as given in Table II.

In summary, the numerical simulations of the deterministic model (2.1) show that prevalence of CA- and HA-MRSA among patients and healthcare workers, mean-time to colonization since admission and the hospital environment are impacted by using control strategies such as low-, moderateand high-effectiveness strategies. These strategies are obtained by varying accordingly the dominant parameters obtained from the sensitivity analysis such as hand washing compliance rate $(\eta)$, the health-care workers decontamination rate $\left(\tau_{W}\right)$, environmental contamination rate $\left(\varepsilon_{W}\right)$, the admission rates into the hospital $\left(\Pi_{P}\right)$, isolation rate of patients with CA-MRSA $\left(\omega_{C C H}\right)$, isolation rate of patients with HA-MRSA $\left(\omega_{C H}\right)$, the transmission probability of CA-MRSA per contact with healthcare workers $\left(\beta_{C}\right)$, and the transmission probability of HA-MRSA per contact with health-care workers $\left(\beta_{H}\right)$.

The numerical simulations further shows that prevalence of MRSA among the hospital patients can be curtail using the high-effectiveness strategy. This is followed by the moderate-effectiveness strategy and the low strategy gave the least performance. This result indicates that a moderate strategy will do a fairly good job in controlling but not 
eliminating MRSA from the hospital. However, for the health-care worker, the high-effectiveness strategy is the only strategy that can effectively control and curtail the disease. The performance of the moderate strategy is just as poor as the low strategy. The difference between them is that the prevalence in the moderate strategy slowly reaches $100 \%$ while it quickly reaches $100 \%$ for the loweffective strategy.

\section{STOChastic MODEL}

The deterministic model gives a detailed biological understanding of the disease transmission dynamics and characteristics. However, this cannot capture small variations and fadeout effects in the various sub-populations, in order to determine the disease incidence and persistence [30]. Thus, we simulated the stochastic model developed in Section IV to investigate these random effects.

Figure 6 depicts twenty realizations of the time series of the total number of hospital patients colonized and infected with CA- and HA-MARSA from the stochastic model and the corresponding result from the deterministic model. From the figures, we observed that the population dynamics of the patients fluctuate over time in the stochastic framework. Moreover, the number of colonized and infected patients are different among the twenty realizations (see Table IV).

Using the result of Section $\mathrm{V}$, we further investigate the prevalence of CA- and HA-MARSA among patients when varying the dominant parameters (hand washing compliance rate $(\eta)$, the health-care workers decontamination rate $\left(\tau_{W}\right)$, isolation rate of patients with CA-MRSA $\left(\omega_{C C H}\right)$ and isolation rate of patients with HA-MRSA $\left(\omega_{C H}\right)$. We observed from Figures 7 and 8 that the prevalence among patients decreases with increasing values of these parameters $\left(\eta, \tau_{W}, \omega_{C C H}\right.$ and $\left.\omega_{C H}\right)$. Figures 7 and 8 indicate a gradual increase in the disease prevalence before converging in distribution after 100 days. The disease prevalence does not converge to a stable state; it is a function of the random state of the process and so, it is a random variable which converges in distribution.
Furthermore, Figures 7 and 8 indicate a nonlinear relationship between the disease prevalence and the model interventions (such as $\eta, \tau_{W}, \omega_{C C H}$, and $\left.\omega_{C H}\right)$. In Figure 7(a), the disease prevalence is lowest at the highest values of the hand washing compliance rate $(\eta)$. However, the prevalence of MRSA is highest among the hospital patients in the absence of health-care workers decontamination (i.e., $\tau_{W}=0$, see in Figure 7 (b) $]$ and absence of isolation of colonized patients (i.e., $\omega_{C C H}=\omega_{C H}=0$, see Figures 7(e) and 7(f) . We observe in Figure 8 similar effects of the parameter values for HA-MRSA prevalence among patients.

\section{Discussions AND CONCLUSION}

In this paper, we have designed and studied deterministic and stochastic models of communityacquired and hospital-acquired methicillinresistant staphylococcus aureus transmission in hospital settings. Unique to our model is the incorporation of the isolated class patients with both CA- and HA-MRSA and the contamination of the hospital environment by the health-care workers. Our goal in this study was to determine an effective control strategy that will curtail and possibly eliminate the spread of the bacteria in the hospital setting. Thus, the intervention strategies we analyzed include the hand washing compliance of healthcare workers, the contamination of the environment, the isolation of infected patients, and the natural decay of the contaminants in the hospital environment.

In the deterministic framework, results indicate the disease-free equilibrium of the model is locallyasymptotically stable whenever the associated reproduction number is less than unity and stable otherwise. The implication of this result is that the disease will continue to spread whenever the reproduction is greater than unity. Likewise, these results show that we may expect the disease to die out whenever the reproduction number is less than unity.

Sensitivity analysis was performed using the Latin Hypercube Sampling (LHS) technique and the par- 
F. B. Agusto et al., Modeling of Community and Hospital-acquired Methicillin-resistant ...

\begin{tabular}{||l||llllllllll||}
\hline \hline Realizations & 1 & 2 & 3 & 4 & 5 & 6 & 7 & 8 & 9 & 10 \\
& 11 & 12 & 13 & 14 & 15 & 16 & 17 & 18 & 19 & 20 \\
\hline \hline$C_{C H}$ & 536 & 567 & 613 & 547 & 568 & 552 & 565 & 559 & 598 & 585 \\
& 553 & 565 & 591 & 632 & 588 & 559 & 524 & 583 & 561 & 568 \\
$I_{C H}$ & 141 & 172 & 159 & 161 & 194 & 172 & 168 & 177 & 153 & 169 \\
& 175 & 152 & 161 & 150 & 170 & 169 & 149 & 164 & 137 & 166 \\
$C_{H}$ & 450 & 422 & 442 & 404 & 455 & 426 & 461 & 403 & 420 & 434 \\
& 457 & 404 & 439 & 442 & 455 & 426 & 397 & 404 & 430 & 462 \\
$I_{H}$ & 86 & 57 & 72 & 54 & 64 & 54 & 76 & 61 & 69 & 73 \\
& 63 & 69 & 57 & 51 & 71 & 57 & 66 & 52 & 80 & 70 \\
$Q$ & 317 & 331 & 319 & 317 & 327 & 351 & 325 & 352 & 335 & 322 \\
& 312 & 368 & 339 & 337 & 337 & 340 & 318 & 307 & 343 & 322 \\
\hline \hline
\end{tabular}

TABLE IV

Simulation of Stochastic MOdel 2.1) AT Time $t=400$ USING 20 REALIZATIONS.

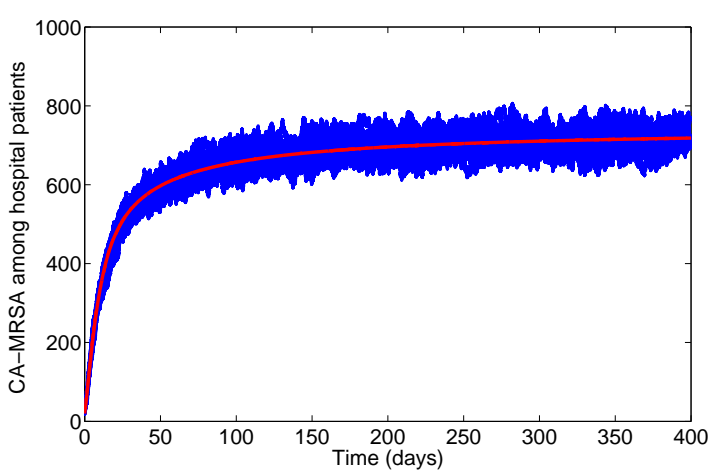

(a)

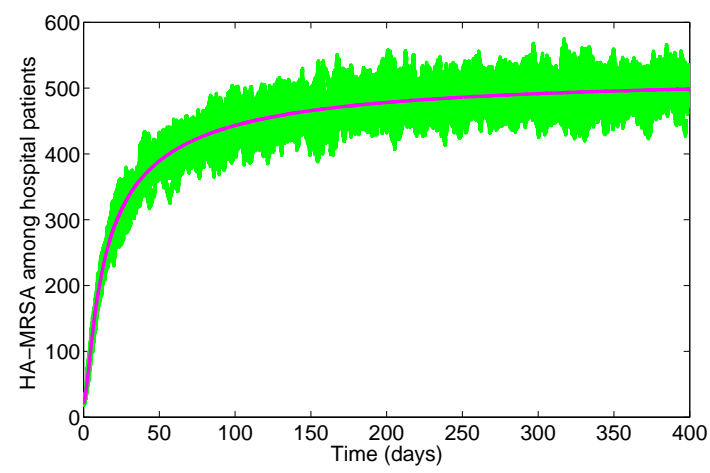

(b)

Fig. 6. Simulation of stochastic model 2.1) as a function of time using 20 realizations. (a). Total number of hospital patients with CA-MRSA; and (b). Total number of hospital patients with HA-MRSA, tial rank correlation coefficient (PRCC) method The LHS stratifies parameters sample space based on the ranges given in Table $\mathrm{II}$ and then sample from these space without replacement. Using response functions the reproduction number $\left(\mathcal{R}_{\mathcal{H C}}\right)$, the prevalence of CA-and HA-MRSA among patients and healthcare workers and the hospital environment, a total of 1,000 simulations of the model (2.1) per LHS run were then carried out and the outcome ranked using PRCC. From Figure 2 and Table III] we conclude that the prevalence of MRSA was the lowest when the rates for hand washing compliance and the bacterial decay were at their highest values. Consequently, the prevalence would increase when the environmental contamination and the transmission rates were high.

In the stochastic framework, we considered a continuous-time Markov chain and assumed the number of health-care workers to be constant. The stochastic process of model 2.1 is shown to be positive recurrent. Using the transitions from the deterministic model 2.1, we derived the instantaneous transition rates for our stochastic model. Numerical simulations of the stochastic model displayed similar results to the deterministic model. We observed an interesting, nonlinear relationship among the prevalence of MRSA among hospital patients and the intervention parameters $\left(\eta, \tau_{W}\right.$, $\omega_{C C H}$, and $\left.\omega_{C H}\right)$. 


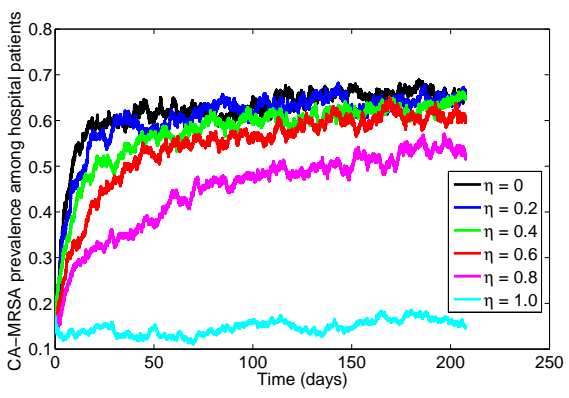

(a)

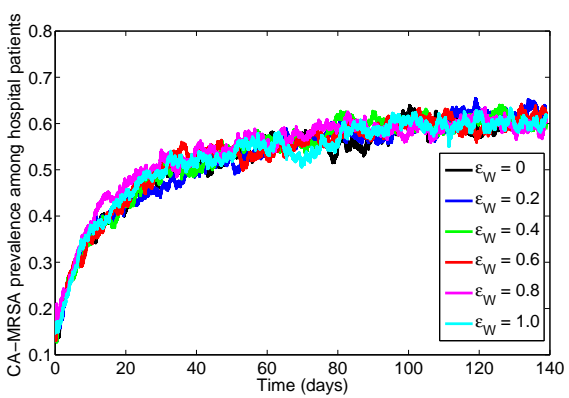

(c)

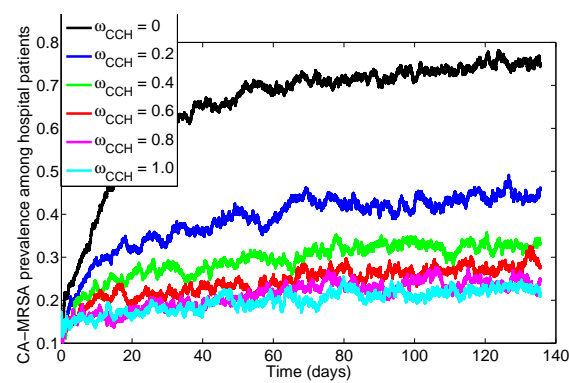

(e)

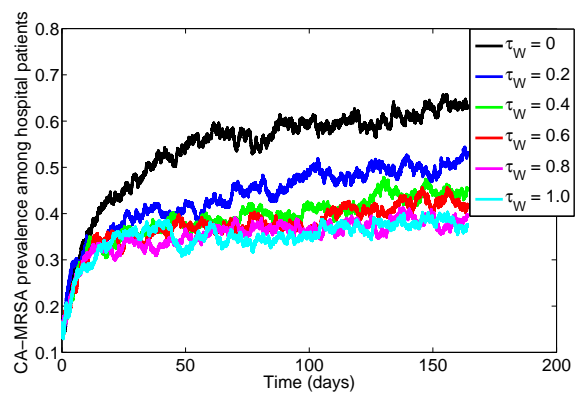

(b)

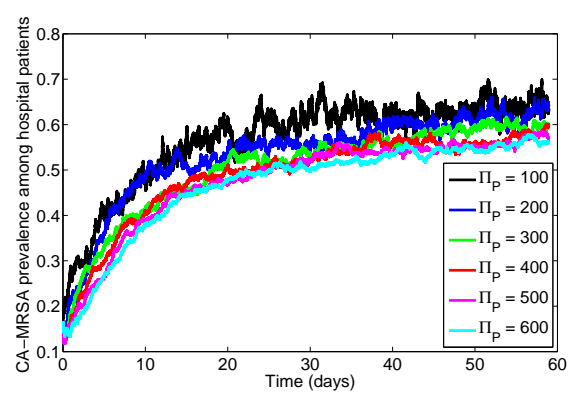

(d)

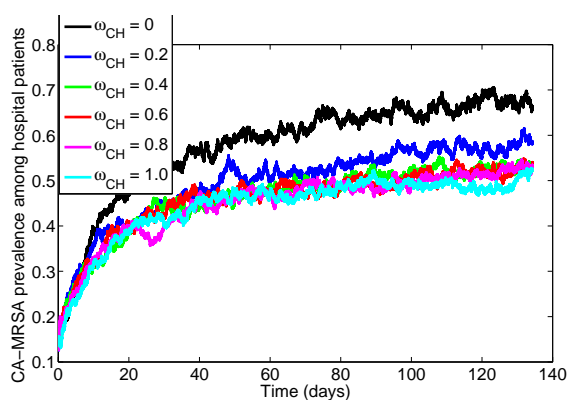

(f)

Fig. 7. Simulation of stochastic model (2.1) using the prevalence of CA-MRSA among hospital patients as model output. Varying: (a). hand washing compliance rate $(\eta)$; (b). health-care workers decontamination rate $\left(\tau_{W}\right)$; (c). environmental contamination rate $\left(\epsilon_{W}\right)$; (d). hospital admission per day $\left(\Pi_{P}\right)$; (e). isolation rate of colonized patients with CA-MRSA $\left(\omega_{C C H}\right)$; (f). isolation rate of colonized patients with HA-MRSA $\left(\omega_{C H}\right)$.

In summary some of the main theoretical and epidemiological findings of this study are summarized below:

(i) The disease-free equilibrium of the model is locally-asymptotically stable whenever the associated reproduction number $\left(\mathcal{R}_{H C}\right)$ is less than unity.

(ii) The disease persists in the community when- ever $\mathcal{R}_{H C}>1$

(iii) The stochastic process of model 2.1 is positive recurrent.

(iv) The sensitivity analysis of the parameter variations using the prevalence rate of CA- and HA-MRSA among patients and health-care workers and the hospital environment as response function the shows the most common 


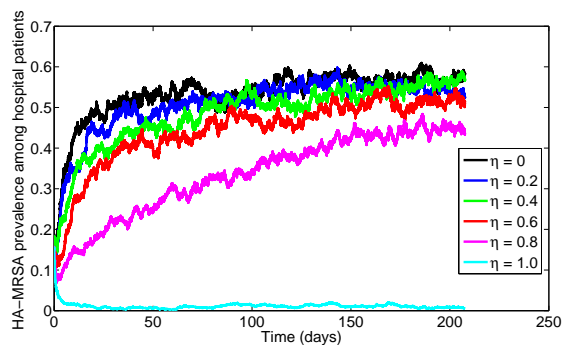

(a)

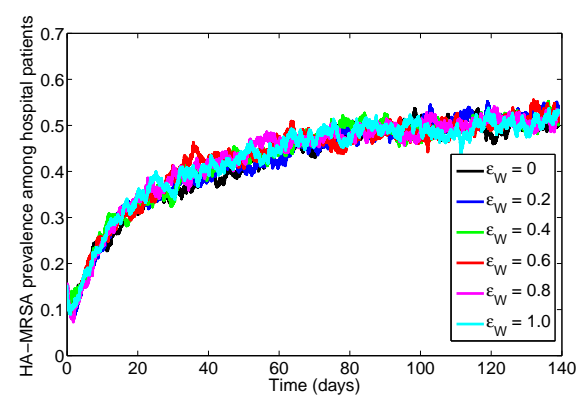

(c)

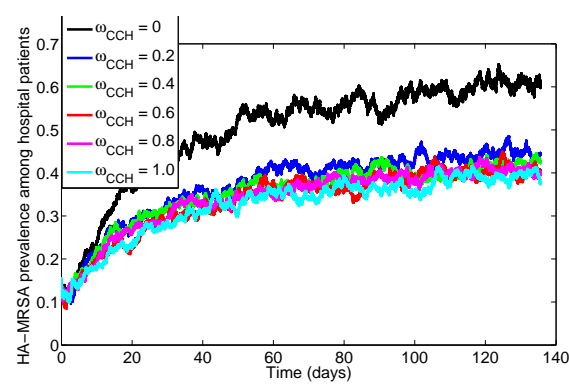

(e)

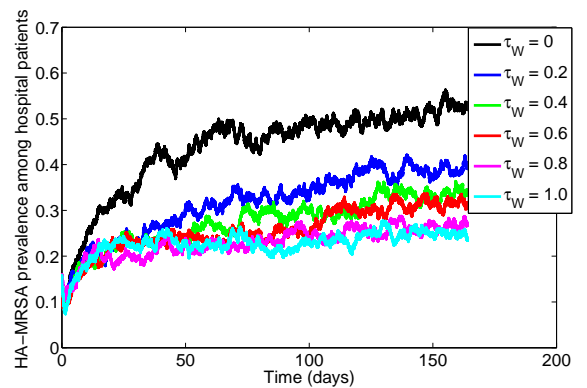

(b)

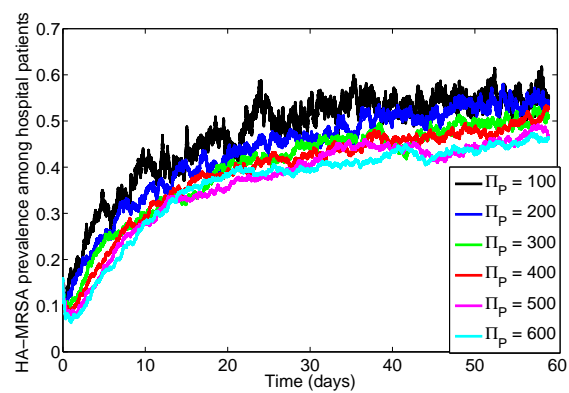

(d)

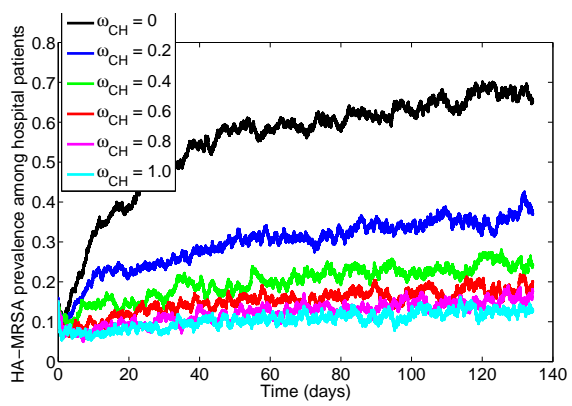

(f)

Fig. 8. Simulation of stochastic model (2.1) using the prevalence of CA-MRSA among hospital patients as model output. Varying: (a). hand washing compliance rate $(\eta)$; (b). health-care workers decontamination rate $\left(\tau_{W}\right) ;($ c). environmental contamination rate $\left(\epsilon_{W}\right)$; (d). hospital admission per day $\left(\Pi_{P}\right)$; (e). isolation rate of colonized patients with CA-MRSA $\left(\omega_{C C H}\right)$; (f). isolation rate of colonized patients with HA-MRSA $\left(\omega_{C H}\right)$.

dominant parameters are the hand washing compliance rate $(\eta)$, the health-care workers decolonization rate $\left(\tau_{W}\right)$, environmental contamination rate $\left(\varepsilon_{W}\right)$, the admission rates into the hospital $\left(\Pi_{P}\right)$, isolation rate of patients with CA-MRSA $\left(\omega_{C C H}\right)$ and isolation rate of patients with HA-MRSA $\left(\omega_{C H}\right)$. In similar dominant are the transmission probabilities of
CA- and HA-MRSA per contact with healthcare workers $\left(\beta_{C}\right.$ and $\left.\beta_{H}\right)$ and transmission probability of health-care workers per contact with patients $\left(\beta_{W}\right)$.

(v) Numerical simulations of both deterministic model (2.1) show that prevalence of CAand HA-MRSA among patients and healthcare workers, mean-time to colonization since 
admission and the hospital environment are impacted by using control strategies such as low-, moderate- and high-effectiveness strategies.

(vi) The prevalence of MRSA among the hospital patients can be curtail using the higheffectiveness strategy.

(a) This result indicates that a moderate strategy will do a fairly good job in controlling but not eliminating MRSA from the hospital;

(b) For the health-care worker, the higheffectiveness strategy is the only strategy that can effectively control and curtail the disease;

(c) On the stochastic frame work, there exist a nonlinear relationship among the prevalence of MRSA among hospital patients and the intervention parameters $\left(\eta, \tau_{W}\right.$, $\omega_{C C H}$, and $\left.\omega_{C H}\right)$.

\section{REFERENCES}

[1] Allen., L.J.S. An introduction to stochastic processes with applications to biology.

[2] Anderson, R.M. and May, R.M. (1991). Infectious Diseases of Humans. Oxford University Press, Oxford.

[3] Blower, S.M. and Dowlatabadi, H. (1994). Sensitivity and uncertainty analysis of complex models of disease transmission: an HIV model, as an example. Int. Stat. Rev., 2: 229-243.

[4] Center for Disease Control (2013). Antibiotic Resistance Threats in the United States, 2013.

[5] Center for Disease Control (2007). Severe methicillinresistant Staphylococcus aureus community-acquired pneumonia associated with influenza - Louisiana and Georgia, December 2006-January 2007. MMWR. 56(14): 325-329. Accessed January 7, 2014.

[6] Chamchod, F. and Ruan,S. (2012). Modeling methicillinresistant Staphylococcus aureus in hospitals: Transmission dynamics, antibiotic usage and its history. Theoretical Biology and Medical Modelling. 9: 25.

[7] Chow, K., Wang, X., Castillo-Chavez, C. (2007). A mathematical model of nosocomial infection and antibiotic resistance: evaluating the efficacy of antimicrobial cycling programs and patient isolation on dual resistance. Arizona State University.

[8] Cookson, B., Bonten, M.J.M., MacKenzie, F.M., Skov, R.L. Verbrugh, H.A. and Tacconelli E. (2011). Meticillinresistant Staphylococcus aureus (MRSA): screening and decolonisation. International Journal of Antimicrobial Agents. 37: 195-201.
[9] Cooper, B.S., Medley, G.F., Stone, S.P., Kibbler, C.C., Cookson, B.D., Roberts, J.A., Duckworth, G., Lai, R. and Ebrahim, S. (2004). Methicillin-resistant Staphylococcus aureus in hospitals and the community: Stealth dynamics and control catastrophes. PNAS. 101(27): 10223-10228.

[10] Cuesta. A, Nastri, N., Bernat, M., Turcot, L., Nastri, M. and Rosa, A.C. (2008). Survival of Staphylococcus aureus on fomites. Acta odontologica latinoamericana : AOL 21(2):141-146.

[11] D'Agata, E., Horn, M., Ruan, S., Webb, G., Wares, J. (2012). Efficacy of infection control interventions in reducing the spread of multidrug-resistant organisms in the hospital setting. PLoSone. 7(2), 1-11.

[12] D'Agata, E.M.C., Webb, G.F. and Pressley, J. (2010). Rapid Emergence of Co-colonization with Communityacquired and Hospital-Acquired Methicillin-Resistant Staphylococcus aureus Strains in the Hospital Setting. Math. Model. Nat. Phenom. 5(3): 76-73.

[13] D'Agata, E., Webb, G., Horn, M., Moellering, Jr., R., Ruan, S. (2009). Modeling the invasion of communityacquired methicillin-resistant stahpylococcus aureus into hospitals. CID. 274-284.

[14] DeLeo, F.R. Otto, M. Kreiswirth, B.N. and Chambers, H.F. (2010). Community-associated meticillin-resistant Staphylococcus aureus. The Lancet. 375: 1557-1568.

[15] Diekmann, O., Heesterbeek, J.A.P. and Metz, J.A.P. (1990). On the definition and computation of the basic reproduction ratio $R_{0}$ in models for infectious diseases in heterogeneous populations. J. Math. Biol. 28: 503-522.

[16] Diep, B.A. anf Otto, M. (2008). The role of virulence determinants in community- associated MRSA pathogenesis. Trends Microbiol. 16:361-369.

[17] Fishbain, J.T., Lee, J.C., Nguyen, H.D., Mikita, J.A., Mikita, C.P., Uyehara, C.F.T., Hospenthal, D.R. (2003). Nosocomial transmission of methicillin-resistant staphylococcus aureus: a blind study to establish baseline acquisition rates. Infect. Cont. Hosp. Ep. f 24: 415-442.

[18] Gehanno, J.F., Louvel, A., Nouvellon, M., Caillard, J.F. and Pestel-Caron, M. (2009). Aerial dispersal of meticillin-resistant Staphylococcus aureus in hospital rooms by infected or colonised patients. J. Hosp. Infect. 71: 256-262.

[19] Girou, E., Loyeau, S., Legrand, P., Oppein, F. and Brun-Buisson, C. (20020. Efficacy of handrubbing with alcohol based solution versus standard handwashing with antiseptic soap: randomised clinical trial. BMJ 325:362.

[20] Gontijo Filho, P.P., Stumpf, M and Cardoso C.L. (1985). Survival of gram-negative and grampositive bacteria artificially applied on the hands. J. Clin. Microbiol. 21(4): $652-653$.

[21] Grundmann, H., Hori, S., Winter, B., Tami, A. and Austin, D.J. (2002). Risk factors for the transmission of methicillin-resistant staphylococcus aureus in an adult intensive care unit: fitting a model to the data. J. Infect. Dis. 185: 481-488.

[22] Hare, R. and Ridley, M. (1958). Further studies on the transmission of Staph. aureus. Br. Med. J. 69-73. 
[23] Herold, B.C., Immergluck, L.C., Maranan, M.C., et al. (1998). Community-acquired methicillin-resistant Staphylococcus aureus in children with no identified predisposing risk. JAMA. 279: 593-598.

[24] Hethcote, H.W. (2000). The mathematics of infectious diseases. SIAM Review. 42(4): 599-653.

[25] Hill J, Howell A, Blowers R. Effect of clothing on dispersal of Staphylococcus aureus by males and females. Lancet 1974; 2:1131-3.

[26] Joyner, M. (2012). Modeling the differences in the development of a new antibiotic class versus the development of a next generation antibiotic on the total resistance in a hospital setting. Journal of Biological Systems. 20(1): 109-132.

[27] Kuehnert, M., Hill, H., Kupronis, B., Tokars, J., Solomon, S., Jernigan, D. (2005). Methicillin-resistant Staphylococcus aureus hospitalizations, United States. Emerg Infect Dis. 11, 868-872.

[28] Kulkarni, V. (1995). Modeling and Analysis of Stochastic Systems. Chapman and Hall.

[29] Lloyd, A.L. Zhang J. and Root, A.M. (2007). Stochasticity and heterogeneity in host-vector models. J. R. Soc. Interface. 4: 851-863 .

[30] Lloyd, A.L. (2004). Estimating variability in models for recurrent epidemics: assessing the use of moment closure techniques. Theoretical Population Biology. 65: 49-65.

[31] Masago, Y., Shibata, T. and Rose, J.B. (2008). Bacteriophage P22 and Staphylococcus aureus attenuation on nonporous fomites as determined by plate assay and quantitative PCR. Appl. Environ. Microbiol. 74:58385840.

[32] Marino, S., Hogue, I.B., Ray, C.J., Kirschner, D.E., (2008). A methodology for performing global uncertainty and sensitivity analysis in systems biology. J. Theor. Biol. 254: 178-196, http://dx.doi.org/10.1016/j.jtbi.2008. 04.011

[33] Mckay, M.D., Beckman, R.J., and Conover, W.J., (1979). Comparison of 3 methods for selecting values of input variables in the analysis of output from a computer code. Technometrics 21: 239-245.

[34] Mediavilla, J.R., Chen, L., Mathema, B. and Kreiswirth, B.N. (2012). Global epidemiology of communityassociated methicillin resistant Staphylococcus aureus (CA-MRSA). Current Opinion in Microbiology. 15: 588595.

[35] Milazzo, L., Brown, J., Eberst, A., Phillips, G., Crawford, J. (2011). Modelling of healthcare associated infections: A study on the dynamics of pathogen transmission by using an individual-based approach. Computer Methods and Programs in Biomedicine. 104, 260-265.

[36] Millar, B. Prendergast, B., Moore, J. (2008). Community-associated MRSA (CA-MRSA): an emerging pathogen in infective endocarditis. J Antimicrob Chemother. 61, 1-7.

[37] Mortimer, E.A., Lipsitz, P.J., Wolinksky, E., Gonzaga, A.J., Rammelkamp, C.H. (1962). Transmission of Staphylococci between newborns: importance of the hands of personnel. Am. J. Dis. Child 104: 289-295.

[38] Otter, J.A. and French, G.L. (2011). Communityassociated meticillin-resistant Staphylococcus aureus strains as a cause of healthcare-associated infection. Journal of Hospital Infection 79 189-193.

[39] Pressley, J., D'Agata, E.M.C. and Webb, G.F. (2010). The effect of co-colonization with community-acquired and hospital-acquired methicillin-resistant Staphylococcus aureus strains on competitive exclusion. Journal of Theoretical Biology. 264: 645-656.

[40] Sanchez, M., Blower, S. (1997). Uncertainty and sensitivity analysis of the basic reproduction rate. American Journal of Epidemiology. 145(12): 1127-1137.

[41] Saravolatz LD, Pohlod DJ, Arking LM. (1982). Community-acquired methicillin-resistant Staphylococcus aureus infections: a new source for nosocomial outbreaks. Ann. Intern. Med. 97: 325-329.

[42] Saravolatz, L.D., Markowitz, N., Arking, L., Pohlod, D., Fisher, E. (1982). Methicillin-resistant Staphylococcus aureus. Epidemiologic observations during a communityacquired outbreak. Ann. Intern. Med. 96: 11-16.

[43] Seybold, U., Kourbatova, E., Johnson, J. (2006). Emergence of community-associated methicillin-resistant Staphylococcus aureus in Los Angeles. New Engl J Med. 352, 1445-1453.

[44] Skov, R.L. Jensen, K.S. (2009). Community-associated meticillin-resistant Staphylococcus aureus as a cause of hospital-acquired infections. Journal of Hospital Infection 73: 364-370.

[45] Thieme, H.R. Mathematics in Population Biology. Princeton University Press, 2003.

[46] Udo, E.E., Pearman J.W. and Grubb, W.B. (1993). Genetic analysis of community isolates of methicillinresistant Staphylococcus aureus in Western Australia. J. Hosp. Infect. 25: 97-108.

[47] Upton, G. and Cook, I. Understanding Statistics. Oxford University Press. 1996. ISBN 0-19-914391-9.

[48] van den Driessche, P. and Watmough, J. (2002). Reproduction numbers and sub-threshold endemic equilibria for compartmental models of disease transmission. Mathematical Biosciences 180: 29-48.

[49] Wolinksky, E, Lipsitz, P.J., Mortimer, E.A., Rammelkamp, C.H. (1960). Acquisition of staphylococci by newborns: direct versus indirect transmission. Lancet. 2: 620-622.

[50] World Health Organization. Drug resistance: Antimicrobial use. http://www.who.int/drugresistance/use/en/. Accessed January 7, 2014.

[51] Zaragoza, M., Salles, M., Gomez, J., Baya,s J.M. and Trilla, A. (1999). Handwashing with soap or alcoholic solutions? A randomized clinical trial of its effectiveness. Am. J. Infect. Control 27: 258-261.

[52] Zwillinger, D. and Kokoska, S. CRC Standard Probability and Statistics Tables and Formulae, CRC Press. 2000. ISBN 1-58488-059-7. 
F. B. Agusto et al., Modeling of Community and Hospital-acquired Methicillin-resistant ...

\section{APPENDIX}

Proof of Lemma 17: It follows from the sum of the first six equations of model (2.1) that

$$
\begin{aligned}
& \frac{d N_{P}(t)}{d t}=\Pi_{P}-\mu_{H} N_{H}(t)-\alpha_{P} U_{P} \\
& -\alpha_{C} C_{C H}-\delta_{C H} I_{H C}(t) \\
& -\alpha_{H} C_{H}-\delta_{H} I_{H}-\left(\delta_{Q}+\alpha_{Q}\right) Q,
\end{aligned}
$$

so that,

$$
\frac{d N_{P}(t)}{d t} \leq \Pi_{P}-\mu_{H} N_{P}(t)
$$

Hence, $\frac{d N_{P}(t)}{d t} \leq 0$ if $N_{P}(0) \geq \frac{\Pi_{P}}{\mu_{H}}$. Thus, $N_{P}(t) \leq N_{P}(0) e^{-\mu_{H} t}+\frac{\Pi_{P}}{\mu_{H}}\left(1-e^{-\mu_{H} t}\right)$. In particular, $N_{P}(t) \leq \frac{\Pi_{P}}{\mu_{H}}$.

From the sum of the equations six and seven of model (2.1) we have

$$
\frac{d N_{W}(t)}{d t}=0
$$

Thus,

$$
N_{W}(t)=K_{W},
$$

where $K_{W}$ is a constant. Hence,

$$
C_{W} \leq N_{W}
$$

From the last equations of model (2.1), we have

$$
\frac{d E_{H}}{d t}=\varepsilon_{W} C_{W}-\rho_{E H} E_{H} .
$$

Using A.3, we have

$$
\frac{d E_{H}}{d t} \leq \varepsilon_{W} K_{W}-\rho_{E H} E_{H} .
$$

Hence, $\frac{d E_{H}}{d t} \leq 0$, if $E_{H}(0) \geq \frac{\varepsilon_{W} K_{W}}{\rho_{E H}}$. Thus, $E_{H}(t) \leq E_{H}(0) e^{-\rho_{E H} t}+\frac{\varepsilon_{W} K_{W}}{\rho_{E H}}\left(1-e^{-\rho_{E H} t}\right)$. In particular $E_{H}(t) \leq \frac{\varepsilon_{W} K_{W}}{\rho_{E H}}$.
Thus, the region $\Omega$ is positively-invariant. Furthermore, if $N_{H}(0)>\frac{\Pi_{H}}{\mu_{H}}$ and $E_{H}(0)>\frac{\varepsilon_{W} K_{W}}{\rho_{E H}}$, then either the solutions enters $\Omega$ in finite time, or $N_{H}(t)$ approaches $\frac{\Pi_{P}}{\mu_{H}}$, with $N_{W}(t)=K_{W}$ and $E_{H}(t)$ approaches $\frac{\varepsilon_{W} K_{W}}{\rho_{E H}}$ asymptotically. Hence, the region $\Omega$ attracts all solutions in $\mathbb{R}_{+}^{9}$.

Proof of Proposition 1

To prove Proposition 1 we need the following lemma.

Lemma 3. Let $\{\mathcal{X}(t), t \geq 0\}$ be a non-negative, vector-valued CTMC on state space

$\Gamma=\left\{x: x_{i} \in\{0,1,2, \ldots\}, i \in\{1, \ldots, n\}\right\}$ with instantaneous transition rates of the form

$$
q_{x, x+e_{i}}=\lambda_{i}, q_{x, x-e_{i}}=\mu_{i} x_{i}, q_{x, x-e_{i}+e_{j}}=\alpha_{i j} x_{i}
$$

with $\lambda_{i}, \alpha_{i j} \geq 0, \mu_{i}>0$ for all $i, j$. Then $\{\mathcal{X}(t), t \geq 0\}$ is positive recurrent.

We will prove Lemma 3 with the following two facts, both of which are proved in [28].

Lemma 4. A CTMC is recurrent (transient) iff its embedded DTMC is recurrent (transient).

Lemma 5. Foster's Criterion. Let $\left\{X_{n}, n \geq 0\right\}$ be an irreducible, discrete-time Markov chain (DTMC) on a countable state space $\Gamma$. The DTMC is positive recurrent if there exists a non-negative function $g$ on $\Gamma$, a finite set $S \subset \Gamma$, and an $\epsilon>0$ such that

$$
\begin{array}{r}
\left|E\left[g\left(X_{n+1}\right)-g\left(X_{n}\right) \mid X_{n}=x\right]\right|<\infty \text { for } x \in S \\
E\left[g\left(X_{n+1}\right)-g\left(X_{n}\right) \mid X_{n}=x\right]<-\epsilon \text { for } x \in S
\end{array}
$$

Proof of Lemma 3. Set $T_{0}=0$ and let $0<$ $T_{1}<T_{2}<\cdots$ denote the transition times of the CTMC $\{\mathcal{X}(t)\}$. Then the embedded DTMC $\left\{\mathcal{X}_{i}, i \in\{0,1,2, \ldots\}\right\}$ is defined through the random vectors $\mathcal{X}_{i}:=\mathcal{X}\left(T_{i}\right)$. Both $\left\{\mathcal{X}_{i}\right\}$ and $\{\mathcal{X}(t)\}$ are irreducible because for any two states $x, y \in \Gamma$, state $y$ is accessible from state $x$.

It remains to show the embedded DTMC $\left\{\mathcal{X}_{i}\right\}$ satisfies Foster's criterion. Take $g(x)=S_{x}:=\sum_{i} x_{i}$ 
F. B. Agusto et al., Modeling of Community and Hospital-acquired Methicillin-resistant ...

in Lemma 5 and define $\beta_{i}:=\sum_{j} \alpha_{i j}$ and

$$
\iota(x):=\sum_{i, j} q_{x, x-e_{i}+e_{j}}=\sum_{i, j} x_{i} \alpha_{i j}=\sum_{i} \beta_{i} x_{i}
$$

We must satisfy the second inequality in Lemma 5 which is

$$
\begin{aligned}
-\epsilon & >E\left[g\left(X_{n+1}\right)-g\left(X_{n}\right) \mid X_{n}=x\right] \\
& =\left(S_{x} \iota(x)+\left(S_{x}+1\right) \sum_{j} \lambda_{j}\right. \\
& \left.+\left(S_{x}-1\right) \sum_{j} \mu_{j} x_{j}\right) /\left(\iota(x)+\sum_{j} \lambda_{j}\right. \\
& \left.+\sum_{j} \mu_{j} x_{j}\right)-S_{x} \\
& =\frac{\sum_{j} \lambda_{j}-\sum_{j} \mu_{j} x_{j}}{\iota(x)+\sum_{j} \lambda_{j}+\sum_{j} \mu_{j} x_{j}}
\end{aligned}
$$

Choose $\epsilon$ small enough so that $\mu_{i}>\epsilon \beta_{i} /(1-\epsilon)$ for each $i$ and set

$$
x_{i}^{*}=\left\lceil\frac{(1+\epsilon) \sum_{k} \lambda_{k}}{\mu_{i}-\epsilon\left(\mu_{i}+\beta_{i}\right)}\right\rceil
$$

and $S=\left\{x \in \Gamma: x_{i}<x_{i}^{*}\right.$ for all $\left.i\right\}$ in Lemma 5. Then for any state $x \in S^{C}$, there exists $i$ such that $x_{i} \geq x_{i}^{*}$, which implies

$$
\frac{1+\epsilon}{1-\epsilon} \sum_{k} \lambda_{k}<x_{i}\left(\mu_{i}-\frac{\epsilon}{1-\epsilon} \beta_{i}\right)
$$

which clearly implies

$$
\frac{1+\epsilon}{1-\epsilon} \sum_{k} \lambda_{k}<\sum_{i} x_{i}\left(\mu_{i}-\frac{\epsilon}{1-\epsilon} \beta_{i}\right)
$$

But this inequality is the same as A.5.

Proof of Proposition 1, The sub-process $\left\{\left(U_{w}(t), C_{w}(t)\right), t \geq 0\right\}$ is trivially positive recurrent because $U_{w}(t)+C_{w}(t)=K_{w}$ for all time $t$. Then the $\left\{E_{h}(t), t \geq 0\right\}$ process is also positive recurrent because the process $\left\{E_{h}^{\prime}(t), t \geq 0\right\}$ with transition rates

$$
\begin{gathered}
q_{e_{h}^{\prime}, e_{h}^{\prime}+1}=K_{w} \varepsilon_{W} \in\left\{0 \leq e_{h}^{\prime} \leq K_{w}-1\right\}, \\
q_{e_{h}^{\prime}, e_{h}^{\prime}-1}=\rho_{E H} e_{h}^{\prime} \in\left\{1 \leq e_{h}^{\prime} \leq K_{w}\right\}
\end{gathered}
$$

is positive recurrent by Lemma 3, and the arrival rate for the primed process bounds that of the original. Finally, all the remaining transition rates of the process in (1) are of the type in Lemma 3 except those involving transitions from compartment $U_{p}$ to $C_{C H}$ or $C_{H}$. However, even if one takes the inter-compartmental transition rates $q_{x, x-e_{u_{p}}+e_{C_{C H}}}$ and $q_{x, x-e_{u_{p}}+e_{C_{H}}}$ to be infinite, the arrival rate of patients from outside the system is a bounded constant and Lemma 3 implies positive recurrence for (1). 